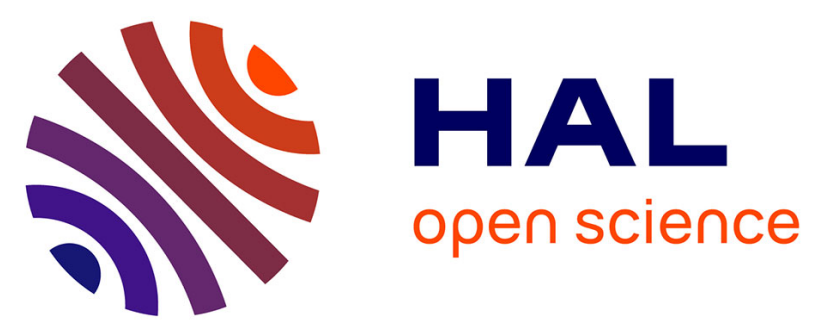

\title{
Mechanistic explanation of the (up to) 3 release phases of PLGA microparticles: Diprophylline dispersions
}

F. Tamani, C. Bassand, M. Hamoudi-Ben Yelles, F. Danède, Jean-François

Willart, F. Siepmann, J. Siepmann

\section{- To cite this version:}

F. Tamani, C. Bassand, M. Hamoudi-Ben Yelles, F. Danède, Jean-François Willart, et al.. Mechanistic explanation of the (up to) 3 release phases of PLGA microparticles: Diprophylline dispersions. International Journal of Pharmaceutics, 2019, pp.118819. 10.1016/j.ijpharm.2019.118819 . hal-02363521

\section{HAL Id: hal-02363521 \\ https://hal.science/hal-02363521}

Submitted on 20 Oct 2020

HAL is a multi-disciplinary open access archive for the deposit and dissemination of scientific research documents, whether they are published or not. The documents may come from teaching and research institutions in France or abroad, or from public or private research centers.
L'archive ouverte pluridisciplinaire $\mathbf{H A L}$, est destinée au dépôt et à la diffusion de documents scientifiques de niveau recherche, publiés ou non, émanant des établissements d'enseignement et de recherche français ou étrangers, des laboratoires publics ou privés. 
Research article

Mechanistic explanation of the (up to) 3 release phases of PLGA microparticles:

Diprophylline dispersions

F. Tamani ${ }^{1}$, C. Bassand ${ }^{1}$, M. Hamoudi-Ben Yelles ${ }^{1}$, F. Danede ${ }^{2}$, J.F. Willart ${ }^{2}$, F. Siepmann ${ }^{1}$, J. Siepmann ${ }^{1, *}$

${ }^{1}$ Univ. Lille, Inserm, CHU Lille, U1008, F-59000 Lille, France

${ }^{2}$ Univ. Lille, USTL UMET UMR CNRS 8207, F-59650 Villeneuve d'Ascq, France

*correspondence:

Prof. Juergen SIEPMANN

University of Lille

College of Pharmacy, INSERM U1008

3 rue du Professeur Laguesse

59006 Lille, France

Phone: +33-3-20964708

juergen.siepmann@univ-lille.fr 


\section{Abstract}

The aim of this study was to better understand the root causes for the (up to) 3 drug release phases observed with poly (lactic-co-glycolic acid) (PLGA) microparticles containing diprophylline particles: The $1^{\text {st }}$ release phase ("burst release"), $2^{\text {nd }}$ release phase (with an "about constant release rate") and $3^{\text {rd }}$ release phase (which is again rapid and leads to complete drug exhaust). The behavior of single microparticles was monitored upon exposure

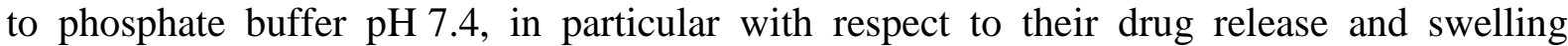
behaviors. Diprophylline-loaded PLGA microparticles were prepared with a solid-in-oil-inwater solvent extraction/evaporation method. Tiny drug crystals were rather homogeneously distributed throughout the polymer matrix after manufacturing. Batches with "small" $(63 \mu \mathrm{m})$, "medium-sized" $(113 \mu \mathrm{m})$ and "large" $(296 \mu \mathrm{m})$ microparticles with a practical drug loading of 5-7 \% were prepared. Importantly, each microparticle releases the drug "in its own way", depending on the exact distribution of the tiny drug crystals within the system. During the burst release, drug crystals with direct surface access rapidly dissolve. During the $2^{\text {nd }}$ release phase tiny drug crystals (often) located in surface near regions which undergo swelling, are likely released. During the $3^{\text {rd }}$ release phase, the entire microparticle undergoes substantial swelling. This results in high quantities of water present throughout the system, which becomes "gel-like". Consequently, the drug crystals dissolve, and the dissolved drug molecules rather rapidly diffuse through the highly swollen polymer gel.

Keywords: PLGA microparticle; release mechanism; burst effect; dissolution; diffusion; swelling. 


\section{Introduction}

Poly (lactic-co-glycolic acid) (PLGA)-based microparticles are frequently used to control drug release upon parenteral administration, because they are completely biodegradable (Vert et al., 1994), biocompatible (Anderson and Shive, 1997), and allow for the adjustment of desired drug release rates during rather flexible periods of time (Johansen et al., 1998; Sheikh Hasan et al., 2015; Bragagni et al., 2018; Qutachi et al., 2018; Riehl et al., 2018). Different types of manufacturing procedures can be used to prepare this type of advanced drug delivery systems, for example solvent extraction/evaporation techniques (Jeffery et al., 1991; Katou et al., 2008; Rawat and Burgess, 2010; Ramazani et al., 2016; Busatto et al., 2018) and spray drying (Wan et al., 2013; Wan and Yang, 2016; Arrighi et al., 2019). The basic idea is to trap the drug within the polymer matrix to avoid instantaneous drug release upon injection into living tissue. The drug can be molecularly dissolved in the PLGA matrix and/or dispersed in the form of tiny (crystalline or amorphous) particles.

The resulting drug release kinetics from PLGA microparticles can be mono-phasic, biphasic or tri-phasic (Friess and Schlapp, 2002; Jiang et al., 2005; Luan and Bodmeier, 2006a; Zolnik and Burgess, 2006; Berkland et al., 2007; Zolnik and Burgess, 2007,2008; Hamishehkar, et al., 2009; Acharya et al., 2010; Awwad et al., 2017; Bragagni et al., 2018; Mylonaki et al., 2018). The $1^{\text {st }}$ release phase is more or less pronounced and also called "burst effect": The drug is rapidly released (generally during several hours or up to 1-2 days) upon contact with aqueous fluids. This phase can be followed by a $2^{\text {nd }}$ release phase with an about constant drug release rate. This "zero order release phase" is variable in length (depending on the type of polymer, e.g. polymer molecular weight) and can take several days or weeks. The slope of the release curve (= the release rate) can be more or less steep. Eventually, drug release is close to negligible (e.g., this part of the release curves looks like a "plateau"). Afterwards, a $3^{\text {rd }}$ release phase might be observed: In these cases, the release rate increases again at a later time point, leading to complete drug exhaust. Not all types of PLGA 
microparticles show drug release profiles exhibiting all 3 phases (some are only mono-phasic, others only bi-phasic). Also, the relative importance of these phases can significantly vary between different types of microparticles. Key factors influencing the shape of release profiles include the type of drug and initial drug loading, the type of PLGA (e.g. initial polymer molecular weight, type of end groups and "lactic acid: glycolic acid" ratio) as well as the manufacturing procedure (affecting the inner and outer structure of the systems). Since important initial burst release might be undesired, different strategies have been described to limit drug release at this stage, e.g. the variation of the manufacturing conditions (Geze et al., 1999; Luan et al., 2006b), incorporation into an outer cubic phase forming in-situ (Ahmed et al., 2008) or the addition of an outer coating (e.g. Huang and Brazel, 2001).

The mass transport mechanisms controlling drug release from PLGA microparticles can be rather complex, including different types of physico-chemical phenomena (Siepmann and Göpferich, 2001; Siepmann and Siepmann, 2008; Fredenberg et al., 2011), such as water penetration into the system, drug dissolution (Siepmann and Siepmann, 2013), drug diffusion (Siepmann and Siepmann, 2012) through water-filled pores, swollen PLGA gel and/or slightly hydrated polymer networks, polyester hydrolysis (Grizzi et al., 1995), drug - PLGA interactions (Park, 1994; Blasi et al., 2007), the creation of water-soluble monomers and oligomers and the latter's diffusion into the surrounding bulk fluid, PLGA swelling, the creation of acidic micro-environments within the microparticles (especially at the center) (Fu et al., 2000; Ibrahim et al., 2005; Schaedlich et al., 2014), resulting in accelerated PLGA degradation (autocatalysis) (Grizzi et al., 1995; Lu et al., 1999; Klose et al., 2006), pore closure effects (Kang and Schwendeman, 2007) and osmotic effects due to the presence of water-soluble compounds within the systems (Brunner et al., 1999), to mention just a few. A comprehensive review on these mechanisms has been given by the group of A. Axelsson (Fredenberg et al., 2011). 
It has to be pointed out that in practice numerous microparticles are administered at the same time and that the resulting drug release kinetics from these ensembles of microparticles are the sums of all the individual drug release profiles from the various single microparticles. For other types of multiple unit dosage forms, e.g. polymer coated controlled release pellets, it has been shown that the release behavior of the single dose units can be very different (e.g., Borgquist et al., 2004; Nevsten et al., 2005). This is not visible when only looking at the drug release kinetics observed with the ensembles of the dosage forms. The monitoring of drug release from single dose units can be very helpful to better understand how these systems work.

Recently, Gasmi et al. (2015a,b, 2016) reported that substantial system swelling (observed with single microparticles) coincided with the onset of the $3^{\text {rd }}$ release phase in different types of PLGA microparticles, loaded with ketoprofen, prilocaine and dexamethasone. Also, Bode et al. $(2019 \mathrm{a}, \mathrm{b})$ reported that the onset of dexamethasone release from macroscopic, hot melt extruded PLGA implants coincided with substantial system swelling. This was true for different "lactic acid: glycolic acid ratios", as well as for poly(lactic acid) (PLA)-based implants, and was explained as follows: At early time points, the polymer chains are relatively long, thus, rather hydrophobic and highly entangled. This limits the amounts of water, which can penetrate into the system. However, some water enters rather rapidly and wets the entire microparticles/implants. This leads to polymer hydrolysis occurring throughout the systems ("bulk erosion") (Von Burkersroda et al., 2002). With time, the polymer chains decrease in length, thus, the degree of macromolecular entanglement decreases and the network becomes mechanically less stable. In addition, ester bond hydrolysis creates $-\mathrm{OH}$ and $-\mathrm{COOH}$ end groups, thus, the system becomes more hydrophilic. Also, the generated monomers and short chain oligomers are water-soluble, creating a steadily increasing osmotic pressure within the system. At a certain time point, substantial amounts of 
water penetrate into the devices, allowing for drug dissolution and facilitated diffusion out into the surrounding bulk fluid.

However, yet the root causes for the $1^{\text {st }}$ and $2^{\text {nd }}$ release phases are less well understood. It is likely that surface near drug contributes to initial burst effects, but details are often unclear and suggested potential reasons for the $2^{\text {nd }}$ release phase are often not based on experimental evidence. The aim of this study was to gain further insight into the mass transport mechanisms controlling drug release from PLGA microparticles, especially during the burst release phase and subsequent about constant drug release phase. For this reason, the behavior of single microparticles loaded with tiny diprophylline crystals was monitored upon exposure to phosphate buffer $\mathrm{pH} 7.4$ using optical and scanning electron microscopy, X-ray powder diffraction, differential scanning calorimetry, in vitro drug release measurements, monitoring of dynamic changes in the microparticles' wet mass as well as energy dispersive X-ray spectrometry.

\section{Materials and methods}

\subsection{Materials}

Poly (D,L lactic-co-glycolic acid) (PLGA; Resomer RG 504H; 50:50 lactic acide:glycolic acid; Evonik, Darmstadt; Germany); diprophylline (BASF, Ludwigshafen, Germany); polyvinyl alcohol (Mowiol 4-88; Sigma-Aldrich, Steinheim, Germany); acetonitrile and dichloromethane (VWR, Fontenoy-sous-Bois, France); tetrahydrofuran (HPLC grade; Fisher Scientific, Illkirch, France).

\subsection{Microparticle preparation}


Drug-loaded microparticles were prepared using a solid-in-oil-in-water (S/O/W) solvent extraction/evaporation technique: Appropriate amounts of diprophylline and PLGA were dispersed/dissolved (the drug was at least partially dispersed in the form of tiny particles, the polymer was dissolved) in a well-defined volume of dichloromethane (Table 1). "Small", "medium-sized" and "large" microparticles were prepared, adapting the formulation and processing parameters accordingly (Table 1). The organic phase was emulsified into 2.5 $\mathrm{L}$ of an outer aqueous polyvinyl alcohol solution $(0.25 \% \mathrm{w} / \mathrm{w})$ under stirring $(1000,1500$ or $2000 \mathrm{rpm}$, Eurostar power-b; Ika-Werke, Staufen, Germany) for $30 \mathrm{~min}$. Upon solvent exchange the PLGA precipitated, trapping the drug. The formed microparticles were hardened by adding $2.5 \mathrm{~L}$ of the same outer aqueous polyvinyl alcohol solution $(0.25 \%)$ and further stirring at $700 \mathrm{rpm}$ (Eurostar power-b) for $4 \mathrm{~h}$. The microparticles were separated by filtration (Nylon filter, $0.45 \mu \mathrm{m}, 13 \mathrm{~mm}$; GE Healthcare Life Sciences Whatman, Kent, UK), washed with de-mineralized water and subsequently freeze-dried (freezing at $-45^{\circ} \mathrm{C}$ for $1 \mathrm{~h} 45 \mathrm{~min}$, primary drying at $-40^{\circ} \mathrm{C}$ and 0.07 mbar for $35 \mathrm{~h}$, and secondary drying at $+20^{\circ} \mathrm{C}$ and 0.0014 mbar for 35 h) (Christ Epsilon 2-4 LSC+; Martin Christ, Osterode, Germany).

\subsection{Microparticle characterization}

\subsubsection{Microparticle size}

Microparticle sizes were determined by optical microscopy: Microscopic pictures were taken using an Axiovision Zeiss Scope-A1 microscope, equipped with an AxioCam ICc1 camera and the Axiovision Zeiss Software (Carl Zeiss, Jena, Germany). For ensembles of microparticles, each measurement included 200 particles. Mean values $+/-$ standard deviations are reported.

\subsubsection{Practical drug loading}


The practical drug loading was determined by dissolving approximately $5 \mathrm{mg}$ microparticles in $5 \mathrm{~mL}$ acetonitrile, followed by filtration (PTFE syringe filters, $0.45 \mu \mathrm{m}$; GE Healthcare, Kent, UK). The drug content was determined by HPLC analysis [Thermo Fisher Scientific Ultimate 3000 Series HPLC, equipped with a LPG 3400 SD/RS pump, an auto sampler (WPS-3000 SL) and a UV-Vis detector (VWD-3400RS); Thermo Fisher Scientific, Waltham, USA]. A reversed phase column Polar C18 (Luna Omega $3 \mu \mathrm{m} ; 150$ x 4.6 mm; Phenomenex, Le Pecq, France) was used. The mobile phase was a mixture of acetate buffer (0.01 M, pH 4.5): acetonitrile (65:35, v:v). The detection wavelength was $274 \mathrm{~nm}$ and the flow rate $1 \mathrm{~mL} / \mathrm{min}$. Five $\mu \mathrm{L}$ samples were injected. The standard curve covered the range of 0.1 to $50 \mu \mathrm{g} / \mathrm{mL}$. Each experiment was conducted in triplicate. Mean values $+/-$ standard deviations are reported.

\subsubsection{X-ray powder diffraction}

X-ray powder diffraction analysis was performed with a Panalytical X'pert pro diffractometer $(\lambda \mathrm{Cu} \mathrm{K \alpha}=1.54 \AA$ ) and Lindemann glass capillaries (diameter $0.7 \mathrm{~mm}$ ) (Panalytical, Almelo, Netherland). The measurements were conducted in transmission mode with an incident beam parabolic mirror and the $\mathrm{X}$ 'celerator detector.

\subsubsection{Differential scanning calorimetry (DSC)}

DSC thermograms of raw materials (as received: diprophylline, PLGA) and of microparticles were recorded with a DSC1 Star System (Mettler Toledo, Greifensee, Switzerland). Approximately $5 \mathrm{mg}$ samples were heated in sealed aluminum pans from $10{ }^{\circ} \mathrm{C}$ to $120^{\circ} \mathrm{C}$, cooled to $-70{ }^{\circ} \mathrm{C}$ and reheated to $120^{\circ} \mathrm{C}$ at a rate of $10^{\circ} \mathrm{C} / \mathrm{min}$. The indicated glass temperatures (Tgs) were obtained from the second heating cycles. Each experiment was conducted in triplicate. Mean values +/- standard deviations are reported. 


\subsubsection{Drug release measurements from ensembles of microparticle}

Ten mg microparticle samples were placed into plastic tubes (Safe-lock tubes $2.0 \mathrm{~mL}$, Eppendorf, Hamburg, Germany) filled with $2 \mathrm{~mL}$ phosphate buffer pH 7.4 (USP 42). The tubes were placed into a horizontal shaker $\left(37^{\circ} \mathrm{C}, 80 \mathrm{rpm}\right.$, GFL 3033; Gesellschaft fuer Labortechnik, Burgwedel, Germany). At predetermined time points, $1.5 \mathrm{~mL}$ samples were withdrawn (replaced with fresh medium), filtered (PTFE syringe filters, $0.45 \mu \mathrm{m}$; GE Healthcare) and analysed for their drug contents by HPLC analysis, as described above. Each experiment was conducted in triplicate. Mean values $+/-$ standard deviations are reported. Sink conditions were provided throughout the experiments.

\subsubsection{Drug release measurements from single microparticles}

Diprophylline release from single microparticles was monitored in 96- well standard microplates (Tissue culture plate 96 well; Carl Roth, Karlsruhe, Germany) as follows: One microparticle was introduced into each well, which was filled with $100 \mu \mathrm{L}$ phosphate buffer pH 7.4 (USP 42) and closed with a cap (Simport Scientific, Beloeil, Quebec). The well microplates were placed into a horizontal shaker $\left(37^{\circ} \mathrm{C}, 80 \mathrm{rpm}\right.$, GFL 3033). At predetermined time points, $50 \mu \mathrm{L}$ samples were withdrawn (replaced with fresh medium) using a Hamilton syringe (Microlite \#710, $100 \mu \mathrm{L}$; Hamilton, Bonaduz, Switzerland) and analysed for their drug contents by HPLC, as described above (however, in this case the standard curve covered the range of 0.025 to $5 \mu \mathrm{g} / \mathrm{mL}$ ).

\subsubsection{Swelling of single microparticles}

Microparticles were treated as for the drug release studies from single microparticles. At pre-determined time points, pictures were taken using an Axiovision Zeiss Scope-A1 microscope and the Axiovision Zeiss Software (Carl Zeiss). The diameter of the microparticles was determined before and after exposure to the release medium (as indicated). 
Furthermore, dynamic changes in the microparticles' wet mass were determined as a function of the exposure time to the release medium as follows: At predetermined time points, samples were carefully withdrawn and excess water removed using Kimtech precision wipes (Kimberly-Clark, Rouen, France). The microparticles' wet mass at time t was measured using an ultra-microbalance (XPR6U; Mettler-Toledo, Greifensee, Switzerland).

\subsubsection{Scanning Electron Microscopy (SEM) and Energy Dispersive X-ray Spectrometry} $(E D S)$

The internal and external morphology of microparticles was studied using a JEOL Field Emission Scanning Electron Microscope (JSM-7800F, Tokyo, Japan), equipped with an EDS microanalysis system (X-Max SDD detector, Aztec 3.3 software; Oxford Instruments, Oxfordshire, England). Samples were fixed with a ribbon carbon double-sided adhesive and covered with a fine chrome layer. Cross-sections were obtained after inclusion of microparticles into "OCT embedding medium" ("embedding medium" for frozen tissue specimen to ensure Optimal Cutting Temperature; VWR BDH, Chemicals, United Kingdom) and cutting with cryostat (Leica CM3050 S, Wetzlar, Germany). Microparticles were observed before and after exposure to the release medium. In the latter case, the microparticles were treated as for the drug release studies from ensembles of microparticles (described above). At predetermined time points, samples were withdrawn, filtered (Nylon, $0.45 \mu \mathrm{m}, 13 \mathrm{~mm}$; GE Healthcare) and freeze-dried (as described above). 


\section{Results and Discussion}

\subsection{Ensembles of microparticles}

Table 2 shows the practical drug loadings, mean particle sizes $(+/-$ standard deviations), glass transition temperatures (Tgs) and optical microscopy pictures of batches of "small", "medium-sized" and "large" PLGA microparticles loaded with diprophylline. This drug was chosen because it is freely water soluble and has a limited solubility in PLGA. The

microparticles were prepared with a solid-in-oil-in-water $(\mathrm{S} / \mathrm{O} / \mathrm{W})$ solvent extraction/evaporation technique: The diprophylline was at least partially dispersed in the form of tiny drug particles within an organic PLGA solution. To obtain differently sized microparticles, the stirring speed as well as the polymer concentration of the organic phase (determining the latter's viscosity) were varied, as indicated in Table 1. Higher stirring speeds and lower organic phase viscosities led to smaller organic droplets and, hence, smaller microparticles. However, also the "surface area : volume" ratio changed and, thus, the degree of drug loss into the outer aqueous phase, resulting in different practical drug loadings. With decreasing droplet size, the latter decreased (data not shown). In order to provide roughly similar practical drug loadings for the differently sized microparticles, the theoretical diprophylline loading was adjusted accordingly (Table 1, by trial-and-error experiments). As it can be seen in Table 2, this resulted in practical drug loadings ranging from $4.8(+/-0.3) \%$ to $6.7(+/-0.4) \%$. It is assumed that the variation of the above mentioned parameters and the minor differences in the drug loading do not fundamentally alter the inner and outer microparticle structure (also, no evidence for noteworthy alterations was observed).

The obtained microparticles were spherical in shape, with mean microparticle sizes of $62.9(+/-19.2), 113.3(+/-40.7)$, and $295.7(+/-94.7) \mu \mathrm{m}$ in the case of "small", "mediumsized" and "large" microparticles, respectively (Table 2). The glass transition temperatures (Tgs) were found to be about $46-47{ }^{\circ} \mathrm{C}$ (Table 2), irrespective of the microparticle diameter. 
This indicates that the PLGA is in the glassy state in the dry microparticles. However, it is well known that small amounts water relatively rapidly penetrate into PLGA-based microparticles (roughly within hours or a day) and that water acts as a plasticizer for this polymer (Faisant et al., 2002; Blasi et al., 2005). Consequently, the Tg of the PLGA in the investigated microparticles can be expected to be below $37^{\circ} \mathrm{C}$ (and the polymer to be in the rubbery state) once the systems are wetted.

Figure 1 shows the resulting drug release kinetics from ensembles of diprophyllineloaded PLGA microparticles in phosphate buffer pH 7.4. Batches with "small", "mediumsized" and "large" microparticles were studied (mean diameters are given). As it can be seen, all three batches exhibited tri-phasic diprophylline release patterns: A burst release $\left(=1^{\text {st }}\right.$ release phase) during the first 1 day (roughly) was followed by a release phase with about constant drug release $\left(=2^{\text {nd }}\right.$ release phase $)$, and a final rapid drug release phase leading to complete drug exhaust $\left(=3^{\text {rd }}\right.$ release phase $)$, which started after about 1 week. The relative importance of the 3 release phases depended on the mean microparticle sizes: The batch with the lowest microparticle size $(63+/-19 \mu \mathrm{m})$ showed an important burst effect $(>50 \%$ drug release) and hardly a $3^{\text {rd }}$ release phase. In contrast, the microparticle batches with "mediumsized" $(113+/-41 \mu \mathrm{m})$ and "large" $(296+/-95 \mu \mathrm{m})$ particles exhibited a much lower burst effect, and a much more pronounced $3^{\text {rd }}$ release phase. The reasons for these differences are discussed below. Please note that, in practice, often microparticles smaller than $100 \mu \mathrm{m}$ are used. However, for technical reasons they are difficult to study individually. The basic assumption in this study is that the internal and external structure of the systems does not fundamentally depend on their size (and no evidence was observed for such differences), so that the underlying mass transport mechanisms controlling drug release are likely the same. Larger microparticles offer the major advantage to allow for the monitoring of the behavior of single microparticles, which can be very helpful to better understand how the systems control drug release. 
Figure 2 shows the X-ray diffraction patterns of ensembles of "small", "mediumsized", and "large" microparticles. For reasons of comparison, also the diffraction patterns of diprophylline powder (as received) is illustrated. Clearly, the drug raw material was crystalline. The sharp diffraction peaks at the same angles observed with the differently sized microparticle batches indicate that diprophylline (at least partially) remained in this crystalline state. This fact can be explained by the manufacturing procedure: A suspension of tiny drug particles in a solution of PLGA in dichloromethane was emulsified into an outer aqueous phase. Upon solvent extraction/evaporation the polymer precipitated and trapped the tiny drug crystals. Thus, the latter did not change their solid state. The top rows in Figures 3 and 4 show representative surfaces and cross-sections of microparticles before exposure to the release medium $(t=0)$. They are representative for all microparticles, irrespective of their size. As it can be seen, tiny drug crystals are randomly and rather homogeneously distributed throughout the PLGA matrix. The surface is relatively smooth and non-porous. Furthermore, the glass transition temperature ( $\mathrm{Tg}$ ) of the PLGA raw material (as received) was found to be equal to $47.0+/-0.2{ }^{\circ} \mathrm{C}$. This is very similar to the Tg values determined for the differently sized microparticle batches $\left(46-47^{\circ} \mathrm{C}\right.$, Table 2$)$. Thus, no noteworthy plasticizing effect of the drug for the polymer was observed. This can serve as an indication for the fact that only minor amounts of the hydrophilic diprophylline are dissolved within the much more hydrophobic polymer. It is likely that major drug portions are dispersed in the form of tiny crystals throughout the systems.

To better understand why the different release phases were observed and why their relative importance depends on the microparticle size, the behavior of single microparticles upon exposure to the release medium was monitored. 


\subsection{Single microparticles}

Optical microscopy pictures of single microparticles, which were exposed to phosphate buffer $\mathrm{pH} 7.4$ at $37^{\circ} \mathrm{C}$ for different time periods are shown in Figures 5 and 6 . Figure 5 covers the entire relevant time period for drug release ( 0 to 17 d). Figure 6 shows additional microscopic pictures of particles after 10 to $28 \mathrm{~d}$ exposure to the release medium, covering the phase of substantial microparticle swelling in more detail. As it can be seen, during the first few days, microparticle swelling was limited, irrespective of the system size. However, after about 1 week exposure to the release medium, substantial microparticle swelling set on. The systems became more and more transparent and "gel like". Please note that each microparticle behaved slightly differently, the swelling was not perfectly homogenous, e.g. little deformations at different locations were observed on a case by case basis. Thus, the environment of a drug crystal ("waiting to be released") varies depending on its exact location. The environment of a particular drug crystal can be expected to strongly affect its release rate.

Figure 7 shows the drug release profiles from single microparticles, together with their swelling kinetics. The red curves refer to the right y-axes, indicating the changes in the particle's diameter. The other (differently colored) curves refer to the left y-axes and illustrate the observed diprophylline release kinetics. The respective (initial) microparticle size is given at the top of each diagram. Interestingly, three types of behaviors can be distinguished:

(i) Certain microparticles do not release any drug prior to the onset of substantial microparticle swelling. The latter occurs after about 1 week, as it can be seen in the diagram in the middle of Figure 8, illustrating the increase in diameter of multiple single microparticles (differing in size) upon exposure to the release medium. Importantly, swelling is limited during the first couple of days, but then becomes very important. The diagram at the bottom of Figure 8 shows the dynamic changes in the wet mass of single PLGA microparticles, which also illustrate the fundamental 
swelling, starting after about 1 week. This coincides with the onset of drug release from certain microparticles shown in Figure 7 (marked by green ovals). This is also the time point at which the $3^{\text {rd }}$ release phase from ensembles of microparticles sets on (final rapid release phase, Figure 1). It has recently been reported that substantial PLGA swelling is likely the root cause for the onset of the $3^{\text {rd }}$ release phase from PLGA microparticles loaded with ketoprofen (Gasmi et al., 2015a), prilocaine (Gasmi et al., 2015b) and dexamethasone (Gasmi et al., 2016). Also, in the case of macroscopic, hot melt extruded, cylindrical implants based on PLGA loaded with dexamethasone the onset of drug release was recently shown to coincide with substantial system swelling (Bode et al., 2019a). The root cause for this type of behavior is likely as follows: At early time points, only limited amounts of water penetrate into the system, since PLGA is rather hydrophobic and the degree of polymer chain entanglement is high (the polymer molecular weight being initially elevated). However, the limited water amounts that can penetrate into the microparticles start degrading the polyester throughout the system ("bulk erosion"). Upon ester bond cleavage, new $-\mathrm{OH}$ and $\mathrm{COOH}$ end groups are created, rendering the system more and more hydrophilic. In addition, the degree of polymer chain entanglement decreases (since the macromolecules become shorter). Also, the generated monomers and oligomers are water soluble and create a steadily increasing osmotic pressure within the microparticles. At a certain time point, the polymeric systems become sufficiently hydrophilic and "mechanically instable" to allow for the penetration of substantial amounts of water into the microparticles: Important microparticle swelling sets on. The penetration of substantial amounts of water into the system fundamentally changes the conditions for the release of the trapped drug crystals: The latter can dissolve in the water and the dissolved drug molecules are rather mobile in the swollen "PLGA gel". Please note that non-dissolved drug cannot diffuse and that limited drug solubility 
effects can be of importance even in the case of freely water-soluble drugs trapped in polymeric controlled drug delivery systems, if the amounts of water available for drug dissolution are limited (Hoang Thi et al., 2017; Siepmann et al., 2017). In addition, the mobility of the dissolved drug molecules is much higher in a highly swollen "PLGA gel" compared to a non-swollen (only slightly hydrated) PLGA matrix. See for example the optical microscopy pictures in the columns on the right hand side versus left hand side in Figures 5 and 6 . This is true, even if the PLGA is in the rubbery state (please see above). Due to the fundamentally facilitated drug dissolution and increased drug mobility, the resulting drug diffusion rate increases and, thus, the release rate increases. This is likely the root cause for the onset of the $3^{\text {rd }}$ drug release phase also in this study. The green region in Figure 8 highlights the respective drug release curves (upper diagram) from single microparticles, which follow this type of behavior. Please note that in the case of the "small" microparticles, this $3^{\text {rd }}$ release phase is not very much pronounced (Figure 1), because most of the drug is already released before the onset of substantial PLGA swelling throughout the system.

It has recently been suggested to call this key role of PLGA swelling for the onset of important drug release "orchestrating role" (in the context of hot melt extruded macroscopic PLGA implants) (Bode et al., 2019a,b). It is the same role that PLGA swelling likely has for the onset of the $3^{\text {rd }}$ drug release phase from microparticles. Figure 9 schematically illustrates this type of drug release behavior at the bottom: "Perfectly" trapped tiny drug crystals "have to wait for their release" until substantial system swelling sets on. Before, the amounts of water getting into contact with these crystals are too small to effectively dissolve them, and the mobility of potentially dissolved drug molecules is rather low in the only slightly hydrated PLGA matrix (even if the latter is in the rubbery state). Please note that different types of drugs likely behave differently, e.g. drugs that easily dissolve in the rubbery PLGA matrix might be 
able to diffuse also through the slightly hydrated polymeric system prior to the onset of substantial PLGA swelling to important extents.

The SEM pictures at the bottom rows in Figures 3 and 4 illustrate how highly swollen "PLGA gels" look like upon freeze-drying (which was required after sampling prior to the SEM measurements): Highly porous structures can be seen and no clear evidence for the presence of drug crystals (because most of the drug is already released after $10 \mathrm{~d}$, Figure 1). Please note that the exact structures that are visible in the SEM pictures are likely artefacts: The highly hydrated PLGA gels probably "collapsed”.

(ii) Other microparticles release at least parts of their drug loading prior to the onset of substantial polymer swelling (before about 1 week in this case). This is marked in red in Figure 7. In certain cases, such "premature" drug release was rather limited (e.g., in the case of the $187 \mu \mathrm{m}$ particle in Figure 7). In other cases, even 2 such "premature release events" were observed (e.g., in the case of the $288 \mu \mathrm{m}$ particle in Figure 7). This behavior can probably be explained as follows: Some of the tiny drug crystals (that are distributed throughout the PLGA microparticles) are relatively close to the systems' surface. Upon exposure to the release medium, microparticle swelling is limited during the first few days (as discussed above), but it is not completely absent. For instance, comparing the 2 columns on the left hand side in Figure 5, showing optical microscopy pictures of microparticles at day 0 (before exposure to the release medium) and day 3, it can be seen that the particles slightly increased in diameter and that the particles' surfaces became less smooth. An example is also illustrated in Figure 10. Thus, the outermost regions of the microparticles become deformed, indicating the swelling of these zones (at least to a certain extent). The overall extent of particle swelling is limited, for instance due to the presence of the still only slightly hydrated and mechanically rather stable inner microparticle core. As long as such a "mechanically stable" core exists, substantial swelling throughout the system is 
hindered. Only once also the core region starts to swell, the microparticle can expand significantly in volume. The limited particle swelling during the first week after exposure to the release medium can also be seen in the diagrams in the middle and at the bottom of Figure 8 (showing dynamic changes in the diameter and wet mass of single microparticles).

If a tiny drug crystal is located close to the microparticle's surface, at a certain time point, this zone likely swells and drug release can set on: The presence of important amounts of water in the direct vicinity of a drug crystal allows for its dissolution and the dissolved drug is rather mobile in the swollen "PLGA gel". As it can be seen in Figure 7, this type of "occasional" drug release event (marked in red) is arbitrary and occurs only in certain microparticles, and at randomly distributed time points. This is because the tiny drug crystals are randomly distributed throughout the systems (Figure 3) and the swelling is not perfectly homogeneous. Each microparticle has "its own" specific inner structure and releases the drug "in its own way" (Figure 7). Adding up all the "occasional" (or "premature") drug release events can likely explain the observed $2^{\text {nd }}$ drug release phase from the ensembles of microparticles (Figure 1). Here, the term "premature" is used to express that this type of drug release occurs prior to the onset of substantial swelling of the entire microparticle (which is the root cause for the onset of the $3^{\text {rd }}$ release phase, as discussed above). Several release curves in the upper diagram in Figure 8 (left to the "green zone") exhibit such "occasional premature release events" (highlighted by small flashes). This local swelling of certain microparticle regions (the likelihood being higher close to the microparticle's surface) might lead to the formation of a more or less homogenous "swelling front", which slowly advances towards the center of the microparticles. Thus, the osberved "occasional"/"premature" drug release events likely occur with an about constant probability over time, explaining the about constant drug release rate in "phase 2". 
Please note that the decrease in surface area of the "swelling front" with time due to the spherical geometry of the system likely only plays a minor role (or is not of importance), because it lasts only about 1 week: Afterwards, substantial microparticle swelling throughout the system becomes dominant (please see above). Figure 9 schematically illustrates this type of drug release behavior ( $2^{\text {nd }}$ release phase).

Please note that the term "swelling front" might be misleading: In the case of macroscopic, cylindrical, hot melt extruded PLGA implants, recently swollen implants "shells" could be distinguished from only slightly hydrated, non-swollen implant cores (Bode et al., 2019b). But these swollen "shells" were not very homogeneous. In the case of microparticles, it is not yet clear how sharp such "swelling fronts" might be. SEM pictures should always be seen with great caution, since system drying prior to the measurements likely creates artefacts. So, the term "swelling front" should be viewed with great caution, it might also be a rather random swelling of certain parts of the PLGA microparticle, not necessarily a clear front that moves inwards.

Further experimental evidence for this release mechanism can be seen in Figure 3: The second row from the top shows SEM pictures of the surfaces of microparticles after $3 \mathrm{~d}$ exposure to the release medium. As it can be seen, crystals are visible on the surface of some particles. Energy dispersive X-ray spectroscopy (EDS) analysis revealed that these crystals are diprophylline crystals (Figure S1, nitrogen being present in the drug, but not in the polymer or other excipients used in this study). Please note that these drug crystals are likely artefacts created during freeze-drying (which was needed to obtain dry samples for the SEM measurements). In the wet state, during drug release, these crystals are very unlikely to exist: The drug is freely water soluble and perfect sink conditions were provided. This drug was likely dissolved either in cavities (formed upon dissolution of drug crystals) or in swollen "PLGA gel" regions ("on its way to diffuse out of the system"). Upon freeze-drying, the drug molecules precipitated and 
formed the needle-shaped crystals at the microparticles' surface (please note the difference in the shape of these crystals compared to the diprophylline crystals embedded within the PLGA matrix before exposure to the release medium, e.g. Figure 4, top row). Importantly, not all microparticles showed this behavior. For example, the surface of the microparticle illustrated in the third row from the top in Figure 3 was free of crystals after $3 \mathrm{~d}$ exposure to the release medium. This highlights the "individuality" of each PLGA microparticle. The second row from the bottom in Figure 3 shows another example for a microparticle with clearly visible drug crystals at its surface, here after $7 \mathrm{~d}$ exposure to the release medium. Again, this is likely due to the precipitation of drug that was dissolved in cavities or in swollen "PLGA gel", contributing to the $2^{\text {nd }}$ release phase. The middle row in Figure 4 shows cross-sections of (freeze-dried) microparticles after $3 \mathrm{~d}$ exposure to the release medium. Importantly, various tiny drug crystals can be seen, the size and shape of which are much more similar to the size and shape of the diprophylline crystals distributed throughout the microparticles prior to exposure to the release medium (Figure 4, top row). Thus, these are likely examples for "well-embedded" drug crystals, which did not come into contact with water prior to the sampling time point (here $3 \mathrm{~d}$ ).

(iii) Other microparticles contain drug crystals, which likely have direct surface access right from the beginning (or very shortly afterwards): In these cases, water can dissolve the drug crystals immediately upon exposure to the release medium, and the drug is rapidly released. The $300 \mu \mathrm{m}$ particle and $352 \mu \mathrm{m}$ particles in Figure 7 are likely examples for such cases. This causes the "burst release" ( $=1^{\text {st }}$ release phase). Figure 9 schematically illustrates this type of drug release behavior. If the surface area in direct contact with the release medium is limited, it can take several hours or eventually days for the entire drug crystal to be released. 
As illustrated in Figure 11a, this phenomenon is much more likely to occur in smaller microparticles than in larger microparticles (if the inner system structures are similar). The same amount of drug is located in numerous small microparticles versus only a few large microparticles (the sums of the volumes of the particle populations being equal). The total number of drug crystals with direct surface access is much higher in the case of the numerous small microparticles compared to the few large microparticles (because the total surface area is much higher; or in other words: the surface area: volume ratio is higher), resulting in a much more pronounced burst effect. This explains why the burst effect was much more important from the ensembles of "small" microparticles compared to the ensembles of "medium-sized" and "large" microparticles, as shown in Figure 1. Please note that it was not possible to monitor the behavior of single microparticles much smaller than about $200 \mu \mathrm{m}$, for technical reasons. Thus, Figure 7 does not show any "small microparticles", which likely show many more "early drug release events", due to drug crystals with direct surface access right from the beginning (or shortly afterwards). It would be interesting to study this aspect with different techniques in the future.

Please also note that the schemes in Figure 9 are simplifications: Drug crystals are illustrated as being individualized, without contact points to other crystals or channels/pores connecting several drug crystals. In reality, at least some of the diprophylline crystals are either directly in contact with each other, or via "channels/pores" (Figure 4, top raw, please note that only 2 dimensional cross-sections are shown, the fact that the crystals are 3dimensional and that right below the visible plane other drug crystals are located, should not be forgotten). Thus, it is likely more realistic that "interconnected networks of drug crystals" exist, as illustrated in Figure 11b: If one of the crystals in such a "network" has "direct surface access" from the beginning ( $1^{\text {st }}$ release phase) or its surrounding swells at a certain time point 
$\left(2^{\text {nd }}\right.$ release phase), also the "connected" drug crystals will likely dissolve and be rather rapidly released afterwards. For reasons of simplicity, this fact is not shown in the other schemes of this article, but it should not be neglected.

Furthermore, please note that in the context of drug release from biodegradable delivery systems, often the term "drug diffusion through pores" is used. Also in this article, we refer to "channels/pores". Looking at the swollen gel structures in Figures 5 and 6, it can be seen that these "gels" are not homogeneous: Their density seems to substantially vary at the micro/nano-scale. Regions with very high water contents might be considered as "pores". However, this term should not be misunderstood: Such "pores" are probably not free of PLGA, at least not at later time points.

\subsection{Drug release mechanisms}

In the following a short summary of the above discussed drug release mechanisms is given:

Importantly, each PLGA microparticle has its own particular inner structure, e.g. with respect to the exact locations of the tiny drug crystals distributed within the PLGA matrix. This individual structure determines whether the microparticle contributes to the $1^{\text {st }}, 2^{\text {nd }}$ and/or $3^{\text {rd }}$ release phases. All options are possible, their likelihood depends among other factors on the microparticle size. The observed release kinetics from ensembles of microparticles (Figure 1) are the sums of all the individual microparticle release behaviors in the sample. As illustrated in Figure 9:

The $1^{\text {st }}$ release phase (burst release) from the investigated PLGA microparticles can likely be attributed to the dissolution of drug crystals with direct surface access right from the beginning (or shortly afterwards) (an example is shown at the top of Figure 12). Drug dissolution is not necessarily instantaneous, but might take up to about $1-2 \mathrm{~d}$, because the drug might have to diffuse through a tiny pore. 
The $2^{\text {nd }}$ release phase (with an about constant drug release rate) is probably caused by the local swelling of certain regions of the PLGA microparticles (with a higher likelihood close to the surface). If a tiny drug crystal is located in such a region, at a certain time point its direct environment undergoes an important change: from a slightly hydrated PLGA matrix to a swollen "PLGA gel". Once this happens, this drug crystal starts dissolving and the dissolved drug molecules are able to diffuse out through the swollen gel (an example is shown in the middle of Figure 12). This type of "release event" occurs occasionally. Since the swelling "front" (please view this term with some caution) likely advances "rather" homogeneously, the probability of these events is about constant over time, resulting in about constant drug release rates.

The $3^{\text {rd }}$ release phase (= final, again rapid drug release phase) is likely caused by substantial PLGA swelling throughout the system: Once the polymer chains are sufficiently hydrophilic and the network becomes "mechanically instable" and no "stable" microparticle core restricts the swelling of the entire system, the osmotic pressure created by the watersoluble degradation products attracts important amounts of water into the microparticles. Consequently, drug dissolution is very much facilitated and the mobility of the dissolved drug molecules significantly increased. Both effects lead to a substantial increase in the drug release rate and finally complete drug exhaust (an example is shown at the bottom of Figure 12). The key role of this substantial PLGA swelling has also been called "orchestrating role", because the swelling determines whether the drug is able to dissolve $\&$ diffuse, or not.

\section{Conclusion}

The aim of this study was to better understand the root causes for the (up to 3) drug release phases of PLGA-based microparticles loaded with drug particles (in particular of the $1^{\text {st }}$ and $2^{\text {nd }}$ release phase). In this case, diprophylline crystals were rather homogeneously 
distributed throughout the polymer matrix after manufacturing. It is suggested that every microparticle has its own, individual inner structure and drug release profile. Each microparticle contributes to one or more drug release phases.

It would be interesting to study other types of microparticles in the future and to use additional experimental measurement techniques to evaluate the validity of the proposed release mechanisms also in other systems. Please note that different drugs can be expected to behave differently. For example, drugs which have a high affinity to PLGA might be able to dissolve to noteworthy extents in only slightly hydrated polymer regions and diffuse through these regions at important rates prior to the onset of substantial microparticle swelling.

\section{Acknowledgements}

This project has received funding from the Interreg 2 Seas programme 2014-2020, cofunded by the European Regional Development Fund under subsidy contract 2S01059_IMODE. The authors are very grateful for this support. They would also like to thank Mr. A. Fadel from the "Centre Commun de Microscopie" of the University of Lille ("Plateau technique de le Federation Chevreul CNRS FR 2638”) as well as Mr. J. Verin and Mr. M. Maton from the INSERM U1008 research group at the University of Lille for their valuable technical help with the SEM pictures.

\section{Conflict of interest}

The Editor-in-Chief of the journal is one of the co-authors of this article. The manuscript has been subject to all of the journal's usual procedures, including peer review, which has been handled independently of the Editor-in-Chief. 


\section{References}

Acharya, G; Shin, CS; Vedantham, K; McDermott, M; Park, K. A study of drug release from homogeneous PLGA microstructures. Journal of Controlled Release, 146, 201-206, 2010.

Ahmed, AR; Dashevsky, A; Bodmeier, R. Reduction in burst release of PLGA microparticles by incorporation into cubic phase-forming systems. European Journal of Pharmaceutics and Biopharmaceutics, 70, 765-769, 2008.

Anderson, JM; Shive, MS. Biodegradation and biocompatibility of PLA and PLGA microspheres. Advanced Drug Delivery Reviews, 28, 5- 24, 1997.

Arrighi, A; Marquette, S; Peerboom, C; Denis, L; Amighi, K. Development of PLGA microparticles with high immunoglobulin G-loaded levels and sustained-release properties obtained by spray-drying a water-in-oil emulsion. International Journal of Pharmaceutics, 566, 291-298, 2019.

Awwad, S; Day, RM; Khaw, PT; Brocchini, S; Fadda, HM. Sustained release ophthalmic dexamethasone: In vitro in vivo correlations derived from the PK-Eye. International Journal of Pharmaceutics, 522, 119-127, 2017.

Berkland, C; Pollauf, E; Raman, C; Silverman, R; Kim, K; Pack, DW. Macromolecule Release from Monodisperse PLG Microspheres: Control of Release Rates and Investigation of Release Mechanism. Journal of Pharmaceutical Sciences, 96, 1176- 1191, 2007.

Blasi, P; D'Souza, SS; Selmin, F; DeLuca, PP. Plasticizing effect of water on poly (lactide-coglycolide). Journal of Controlled Release, 108, 1-9, 2005.

Blasi, P; Schoubben, A; Giovagnoli, S; Perioli, L; Ricci, M; Rossi, C. Ketoprofen poly (lactide-co-glycolide) physical interaction. AAPS PharmSciTech, 8, 78-85, 2007. 
Bode, C; Kranz, H; Fivez, A; Siepmann, F; Siepmann, J. Often neglected: PLGA/PLA swelling orchestrates drug release - HME implants. Journal of Controlled Release, 306, 97-107, 2019a.

Bode, C; Kranz, H; Siepmann, F; Siepmann, J. Coloring of PLGA implants to better understand the underlying drug release mechanisms. International Journal of Pharmaceutics, in press, 2019b.

Borgquist, P; Nevsten, P; Nilsson, B; Wallenberg, LR; Axelsson, A. Simulation of the release from a multiparticulate system validated by single pellet and dose release experiments. Journal of controlled release, 97, 453-465, 2004.

Bragagni, M; Gil-Alegre, ME; Mura,P; Cirri, M; Mannelli, LDC. Improving the therapeutic efficacy of prilocaine by PLGA microparticles: Preparation, characterization and in vivo evaluation. International Journal of Pharmaceutics, 547, 24-30, 2018.

Brunner, A; Mäder, K; Göpferich, A. pH and osmotic pressure inside biodegradable microspheres during erosion. Pharmaceutical research, 16, 847-853, 1999.

Busatto, C; Pesoa, J; Helbling, I; Luna, J; Estenoz, D. Effect of particle size, polydispersity and polymer degradation on progesterone release from PLGA microparticles: Experimental and mathematical modelling. International Journal of Pharmaceutics, $536,360-369,2018$

Faisant, N; Siepmann, J; Benoit, JP. PLGA-based microparticles: Elucidation of mechanisms and a new, simple mathematical model quantifying drug release. European Journal of Pharmaceutical Sciences 15, 355-366, 2002.

Fredenberg, S; Wahlgren, M; Reslow, M; Axelsson, A. The mechanisms of drug release in poly (lactic-co-glycolic acid)-based drug delivery systems-a review. International journal of pharmaceutics, 415, 34-52, 2011.

Friess, W; Schlapp, M. Release mechanisms from gentamicin loaded poly (lactic-co-glycolic acid) (PLGA) microparticles. Journal of Pharmaceutical Sciences, 91, 845- 855, 2002. 
Fu, K; Pack, DW; Klibanov, AM; Langer, R. Visual evidence of acidic environment within degrading poly (lactic-co-glycolic acid) (PLGA) microspheres. Pharmaceutical Research, 17, 100- 106, 2000.

Gasmi, H; Danede, F; Siepmann, J; Siepmann, F. Does PLGA microparticle swelling control drug release? New insight based on single particle swelling studies. Journal of Controlled Release, 213, 120-127, 2015a.

Gasmi, H; Siepmann, F; Hamoudi, MC; Danede, F; Verin, J; Willart, JF; Siepmann, J. Towards a better understanding of the different release phases from PLGA microparticles: Dexamethasone-loaded systems. International Journal of Pharmaceutics, 514, 189-199, 2016.

Gasmi, H; Willart, JF; Danede, F; Hamoudi, MC; Siepmann, J; Siepmann, F. Importance of PLGA microparticle swelling for the control of prilocaine release. Journal of Drug Delivery Science and Technology, 30, 123-132, $2015 \mathrm{~b}$.

Geze, A; Venier-Julienne, MC; Mathieu, D; Filmon, R; Phan-Tan-Luu, R; Benoit, JP. Development of 5-iodo-2'-deoxyuridine milling process to reduce initial burst release from PLGA microparticles. International Journal of Pharmaceutics, 178, 257-268, 1999.

Grizzi, I; Garreau, H; Li, S; Vert, M. Hydrolytic degradation of devices based on poly (dllactic acid) size-dependence. Biomaterials, 16, 305-311, 1995.

Hamishehkar, H; Emami, J; Najafabadi, AR; Gilani, K; Nokhodchi, A. The effect of formulation variables on the characteristics of insulin-loaded poly (lactic-co-glycolic acid) microspheres prepared by a single phase oil in oil solvent evaporation method. Colloids and Surfaces B: Biointerfaces, 74, 340-349, 2009.

Huang, X; Brazel, C. On the importance and mechanisms of burst release in matrix-controlled drug delivery systems. Journal of Controlled Release, 73, 121-136, 2001. 
Hoang Thi, Th; Priemel, PA; Karrout, Y; Driss, V; Delbeke, M; Dendooven, A; Flament, MP; Capron, M; Siepmann, J. Preparation and investigation of P28GST-loaded PLGA microparticles for immunomodulation of experimental colitis. International Journal of Pharmaceutics, 533, 26-33, 2017.

Ibrahim, MA; Ismail, A; Fetouh, MI; Göpferich, A. Stability of insulin during the erosion of poly (lactic acid) and poly (lactic-co-glycolic acid) microspheres. Journal of Controlled Release, 106, 241-252, 2005.

Jeffery, H; Davis, SS; O'Hagan, DT. The preparation and characterisation of poly(lactide-coglycolide) microparticles. I: Oil-in-water emulsion solvent evaporation. International Journal of Pharmaceutics, 77, 169-175, 1991.

Jiang, W; Gupta, RK; Deshpande, MC; Schwendeman, SP. Biodegradable poly (lactic-coglycolic acid) microparticles for injectable delivery of vaccine antigens. Advanced Drug Delivery Reviews, 57, 391-410, 2005.

Johansen, P; Corradin, G; Merkle, HP; Gander, B. Release of tetanus toxoid from adjuvants and PLGA microspheres: How experimental set-up and surface adsorption fool the pattern. Journal of Controlled Release, 56, 209- 217, 1998.

Kang, J; Schwendeman, SP. Pore closing and opening in biodegradable polymers and their effect on the controlled release of proteins. Molecular pharmaceutics, 4, 104-118, 2007.

Katou, H; Wandrey, AJ; Gander, B. Kinetics of solvent extraction/evaporation process for PLGA microparticle fabrication. International Journal of Pharmaceutics, 364, 45-53, 2008.

Klose, D; Siepmann, F; Elkharraz, K; Krenzlin, S; Siepmann, J. How porosity and size affect the drug release mechanisms from PLGA-based microparticles. International journal of pharmaceutics, 314, 198-206, 2006. 
Lu, L; Garcia, CA; Mikos, AG. In vitro degradation of thin poly (DL- lactic- co- glycolic acid) films. Journal of Biomedical Materials Research, 46, 236-244, 1999.

Luan, X; Bodmeier, R. Modification of the tri-phasic drug release pattern of leuprolide acetate-loaded poly (lactide-co-glycolide) microparticles. European Journal of Pharmaceutics and Biopharmaceutics, 63, 205-214, 2006a.

Luan, X; Skupin, M; Siepmann, J; Bodmeier, R. Key parameters affecting the initial release (burst) and encapsulation efficiency of peptide-containing poly(lactide-co-glycolide) microparticles. International Journal of Pharmaceutics, 324, 168-175, $2006 \mathrm{~b}$.

Mylonaki, I; Allémann, E; Delie, F; Jordan, O. Imaging the porous structure in the core of degrading PLGA microparticles: The effect of molecular weight. Journal of Controlled Release, 286, 231-239, 2018.

Nevsten, P; Borgquist, P; Axelsson, A; Wallenberg, LR. XEDS-mapping for explaining release patterns from single pellets. International journal of pharmaceutics, 290, 109120, 2005.

Qutachi, O; Wright, EJ; Bray, G; Hamid, OA; Delcassian, D. Improved delivery of PLGA microparticles and microparticle-cell scaffolds in clinical needle gauges using modified viscosity formulations. International Journal of Pharmaceutics, 546, 272$278,2018$.

Park, TG. Degradation of poly(d,l-lactic acid) microspheres: effect of molecular weight. Journal of Controlled Release, 30, 161- 173, 1994.

Ramazani, F; Weiluan, C; Van Nostrum, CF; Storm, G; Kok, RJ. Strategies for encapsulation of small hydrophilic and amphiphilic drugs in PLGA microspheres: State-of-the-art and challenges. International Journal of Pharmaceutics, 499, 358-367, 2016.

Rawat, A; Burgess DJ. Effect of ethanol as a processing co-solvent on the PLGA microsphere characteristics. International Journal of Pharmaceutics, 394, 99-105, 2010. 
Riehl, M; Harms, M; Lucas, H; Ebensen, T; Mäder, K. Dual dye in-vivo imaging of differentially charged PLGA carriers reveals antigen-depot effect, leading to improved immune responses in preclinical models. European Journal of Pharmaceutical Sciences, 117, 88-97, 2018.

Schädlich, A; Kempe, S; Mäder, K. Non-invasive in vivo characterization of microclimate pH inside in situ forming PLGA implants using multispectral fluorescence imaging. Journal of Controlled Release, 179, 52-62, 2014.

Sheikh Hasan, A; Sapin, A; Damgé, C; Leroy, P; Socha, M; Maincent, P. Reduction of the in vivo burst release of insulin-loaded microparticles. Journal of Drug Delivery Science and Technology, 30, 486- 493, 2015.

Siepmann, J; Göpferich, A. Mathematical modeling of bioerodible, polymeric drug delivery systems. Advanced Drug Delivery Reviews, 48, 229-247, 2001.

Siepmann, F; Karrout, Y; Gehrke, M; Penz, F; Siepmann, J. Limited drug solubility can be decisive even for freely soluble drugs in highly swollen matrix tablets. International Journal of Pharmaceutics, 526, 280-290, 2017.

Siepmann, J; Siepmann, F. Mathematical modeling of drug delivery. International Journal of Pharmaceutics, 364, 328-343, 2008.

Siepmann, J; Siepmann, F. Modeling of diffusion controlled drug delivery. Journal of Controlled Release, 161, 351-362, 2012.

Siepmann, J; Siepmann, F. Mathematical modeling of drug dissolution. International Journal of Pharmaceutics, 453, 12-24, 2013.

Vert, M; Mauduit, J; Li, S. Biodegradation of PLA/GA polymers: increasing complexity. Biomaterials, 15, 1209- 1213, 1994.

Von Burkersroda, F; Schedl, L; Göpferich, A. Why degradable polymers undergo surface erosion or bulk erosion. Biomaterials, 23, 4221-4231, 2002. 
Wan, F; Wu, JX; Bohr, A; Baldursdottir, SG; Yang, M. Impact of PLGA molecular behavior in the feed solution on the drug release kinetics of spray dried microparticles. Polymer, 54, 5920-5927, 2013.

Wan, F; Yang, M. Design of PLGA-based depot delivery systems for biopharmaceuticals prepared by spray drying International. Journal of Pharmaceutics, 498, 82-95, 2016.

Zolnik, BS; Burgess, DJ. Effect of acidic pH on PLGA microsphere degradation and release. Journal of Controlled Release, 122, 338-344, 2007.

Zolnik, BS; Burgess, DJ. Evaluation of in vivo-in vitro release of dexamethasone from PLGA microspheres. Journal of Controlled Release, 127, 137-145, 2008.

Zolnik, BS; Leary, PE; Burgess, DJ. Elevated temperature accelerated release testing of PLGA microspheres. Journal of Controlled Release, 112, 293-300, 2006. 


\section{Table 1:}

Composition of the inner organic phase and stirring speed used for the preparation of "small", "medium-sized" and "large" PLGA microparticles loaded with diprophylline.

\begin{tabular}{ccccc}
\hline Microparticle size & $\mathrm{CH}_{2} \mathrm{Cl}_{2}, \mathrm{~mL}$ & PLGA, mg & Drug, mg & Stirring speed, rpm \\
\hline "Small" & 10 & 900.1 & 204.4 & 2000 \\
"Medium-sized" & 6 & 834.3 & 125.0 & 1500 \\
"Large" & 4 & 909.5 & 101.0 & 1000 \\
\hline
\end{tabular}


Table 2:

Practical drug loadings, mean particle sizes, glass transition temperatures (Tgs) and morphology of "small", "medium-sized" and "large" PLGA microparticles loaded with diprophylline (mean values $+/$ - standard deviations are reported).

Practical loading, $\% \quad$ Mean size, $\mu \mathrm{m}$

$\mathrm{Tg},{ }^{\circ} \mathrm{C}$

Optical microscopy

$\begin{array}{llll}\text { "Small" } & 4.8 \pm 0.3 & 62.9 \pm 19.2 & \\ \text { "Medium-sized" } & 5.8 \pm 0.6 & 113.3 \pm 40.7 & 46.8 \pm 0.1 \\ \text { "Large" } & 295.7 \pm 94.9 & 46.3 \pm 0.3\end{array}$




\section{Figure captions}

Fig. 1: Diprophylline release from ensembles of PLGA microparticles in phosphate buffer $\mathrm{pH}$ 7.4: Impact of the mean particle size (indicated in the diagram $+/-$ standard deviation). The release profiles are tri-phasic: an initial burst release $\left(=1^{\text {st }}\right.$ phase $)$ is followed by a period with an about constant drug release rate $\left(=2^{\text {nd }}\right.$ phase $)$ and a final (again) rapid drug release phase leading to complete drug exhaust $\left(=3^{\text {rd }}\right.$ phase). Please note that the transition periods are not always very sharp. Also, in the case of the "small" microparticles $(62+/-19 \mu$ m diameter $)$, the $3^{\text {rd }}$ release phase is not very pronounced, since most of the drug is already released at this time point. The cartoons indicate the hypothesized drug release mechanisms (details are given in the text).

Fig. 2: X-ray diffraction patterns of ensembles of diprophylline-loaded PLGA microparticles (mean particle sizes +/- standard deviations are indicated in the diagram). For reasons of comparison, also the diffraction patterns of diprophylline powder (as received) is shown.

Fig. 3: SEM pictures of surfaces (lower, medium and higher magnification) of diprophylline-loaded microparticles before and after exposure to phosphate buffer pH 7.4 for different time periods (indicated on the left hand side, two examples are shown for $t=3 d)$. Note that the microparticles were freeze-dried after exposure to the release medium, which likely created artefacts.

Fig. 4: SEM pictures of cross-sections at different magnifications of diprophylline-loaded microparticles before and after exposure to phosphate buffer $\mathrm{pH} 7.4$ for different time periods (indicated on the left hand side). Note that the microparticles were freeze-dried after exposure to the release medium, which likely created artefacts. 
Fig. 5: Optical microscopy pictures of single diprophylline-loaded PLGA microparticles before and after exposure to phosphate buffer $\mathrm{pH} 7.4$ for different time periods (indicated at the top). The initial particle size is given on the left hand side.

Fig. 6: Optical microscopy pictures of single diprophylline-loaded PLGA microparticles after 10-28 d exposure to phosphate buffer $\mathrm{pH}$ 7.4. The initial particle size is given on the left hand side.

Fig. 7: Drug release and swelling of single PLGA microparticles upon exposure to phosphate buffer $\mathrm{pH}$ 7.4. The initial microparticle sizes are indicated at the top of each diagram. "Occasional/premature" drug release is marked in red, drug release following the onset of substantial swelling of the entire system is marked in green.

Fig. 8 Behavior of single PLGA microparticles loaded with diprophylline upon exposure to phosphate buffer $\mathrm{pH}$ 7.4: Drug release, dynamic changes in the diameter and dynamic changes in the wet mass. Each curve corresponds to a single microparticle.

Fig. 9: Schematic illustration of the involved mass transport phenomena controlling diprophylline from the investigated PLGA microparticles during the $1^{\text {st }}, 2^{\text {nd }}$ and $3^{\text {rd }}$ release phase. Non-swollen (only slightly hydrated) PLGA is marked in dark grey, swollen PLGA in light grey. Details are given in the text. Please note that the schemes are simplifications, e.g. with respect to the homogeneity of polymer swelling. Also, each microparticle has a specific, individual inner structure (e.g. location of the trapped drug crystals) and might contribute to 1 or more drug release phases.

Fig. 10 Optical microscopy pictures of a single diprophylline-loaded PLGA microparticle before and after $3 \mathrm{~d}$ exposure to phosphate buffer $\mathrm{pH}$ 7.4.

Fig. 11 a) Schematic presentation of a PLGA microparticle of a larger and several smaller microparticles: If the inner system structure is similar, the likelihood of drug crystals 
with direct surface access is much higher in the case of smaller microparticles, resulting in much more pronounced burst effects. b) Schematic presentation of a PLGA microparticle loaded with tiny drug crystals. The left drawing is a simplification showing only isolated drug crystals. The right drawing is likely much more realistic (at least for the investigated type of microparticles in this study), showing interconnections between some of the tiny drug crystals, forming "networks". Please note that these are 2-dimensional schemes, in reality the microparticles are spherical and interconnected networks are formed in all 3 dimensions.

Fig. 12 Examples for single microparticles contributing to the different phases of drug release from the investigated PLGA microparticles. The experimental results show drug release from and the swelling of the systems, the schemes illustrate the likely root causes for drug release. Details are explained in the text. 


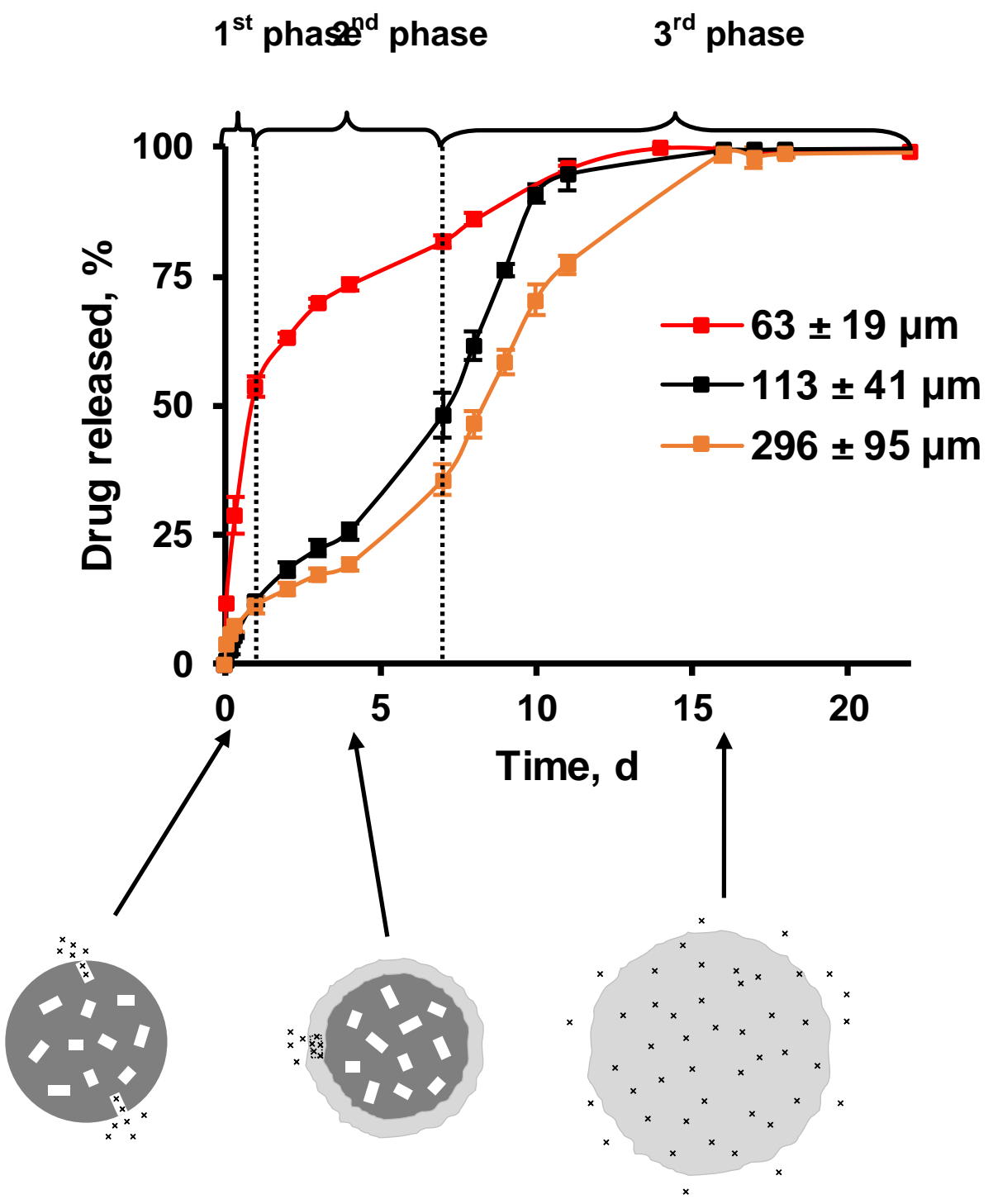

Figure 1 


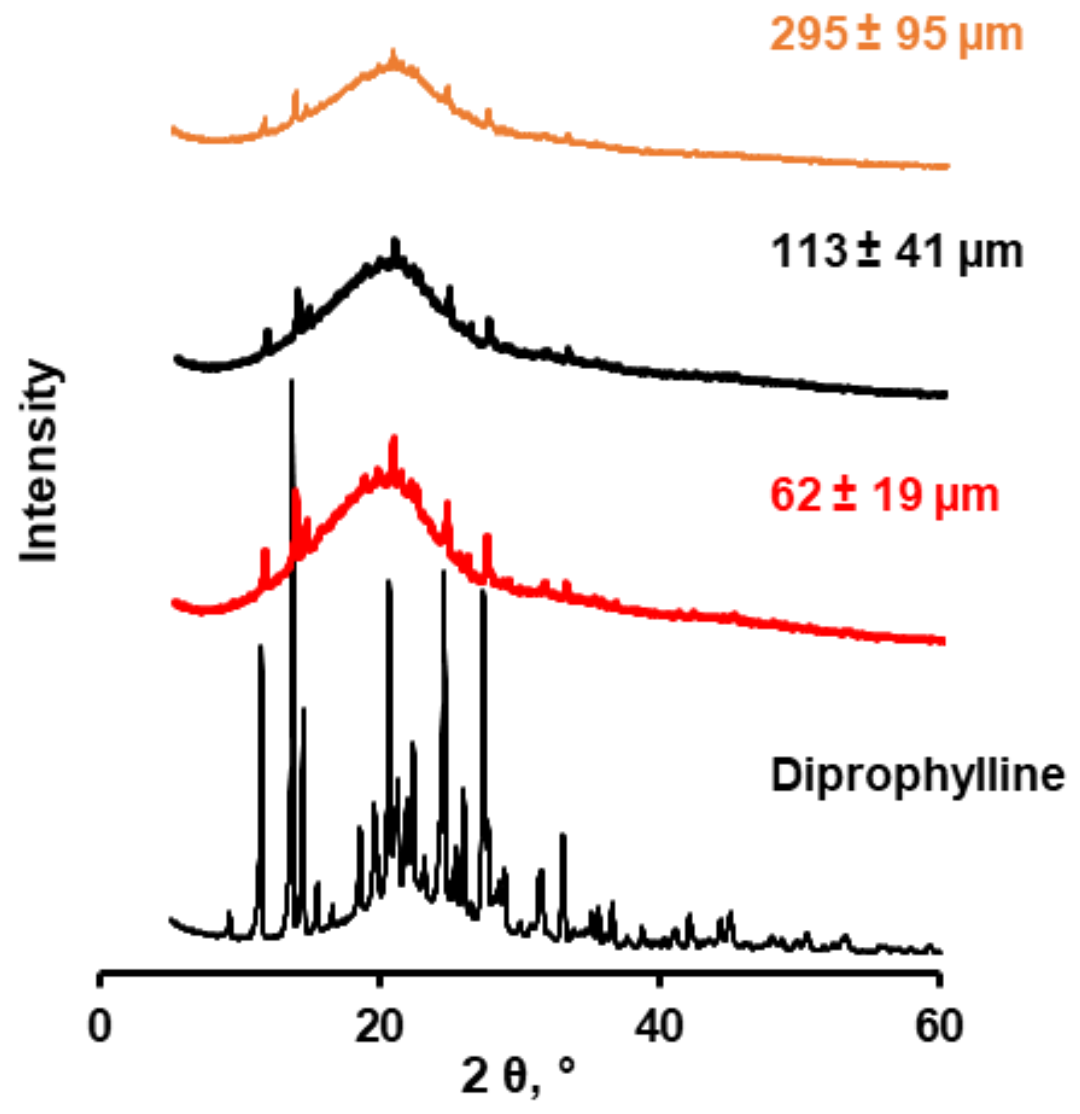

Figure 2 
Lower magnification Medium magnification

Higher magnification
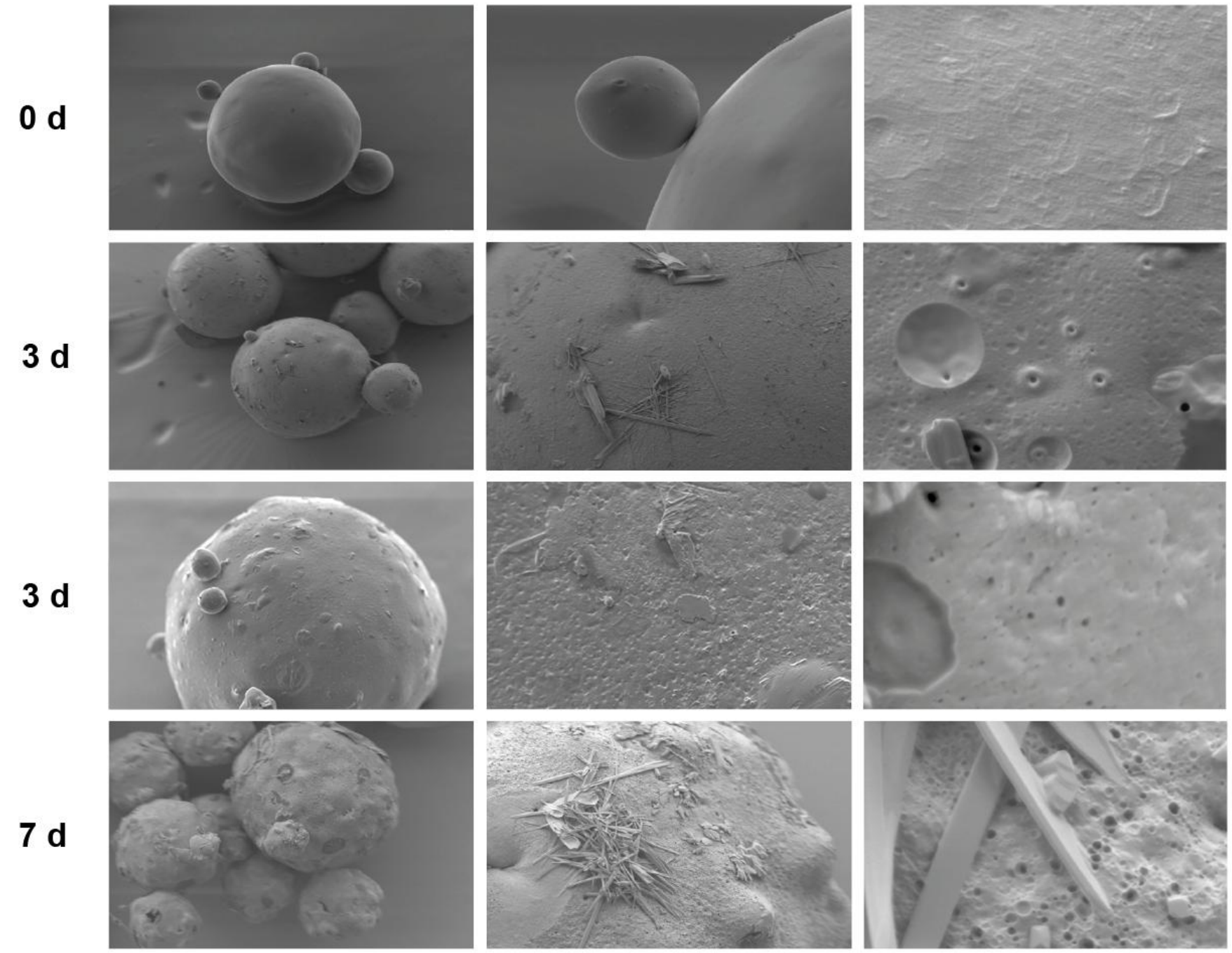

$7 \mathrm{~d}$
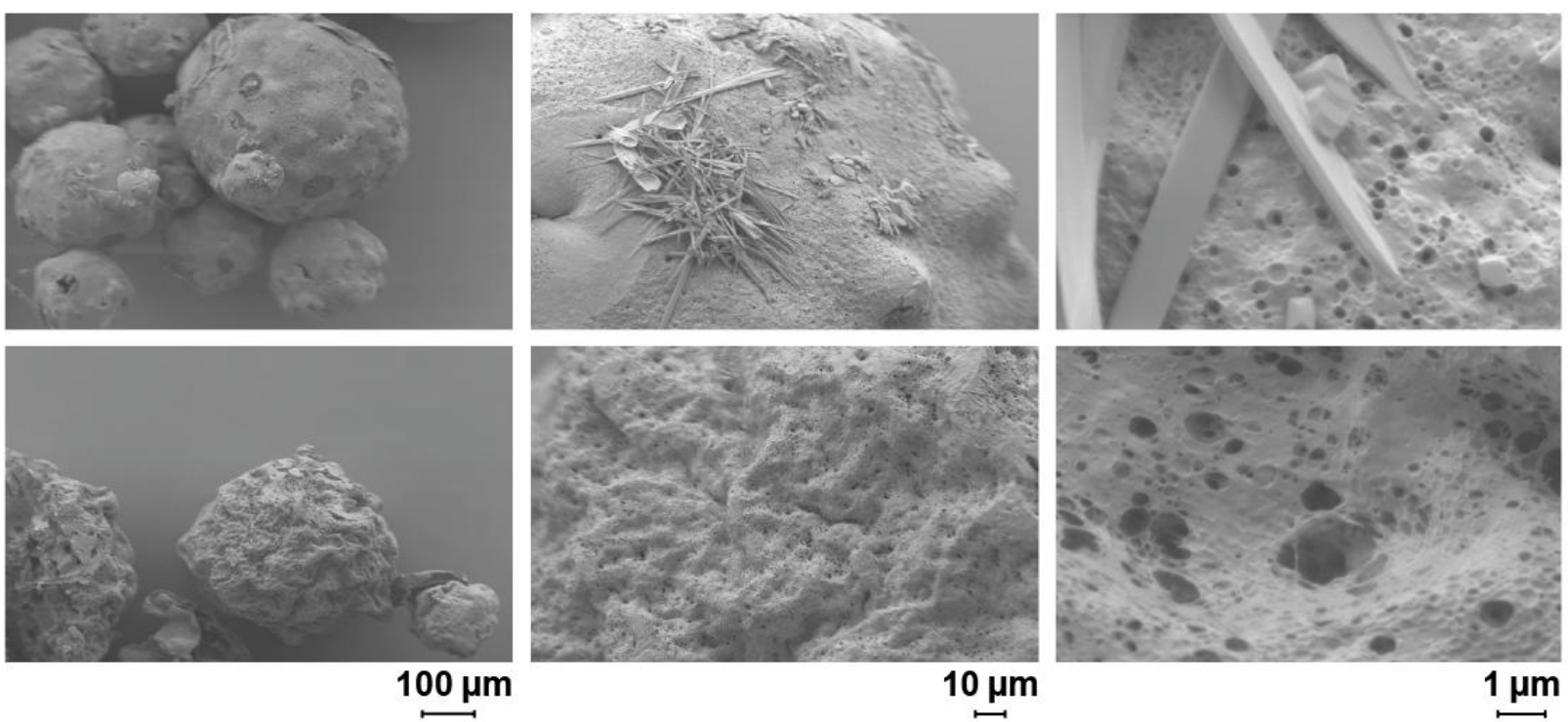

Figure 3 

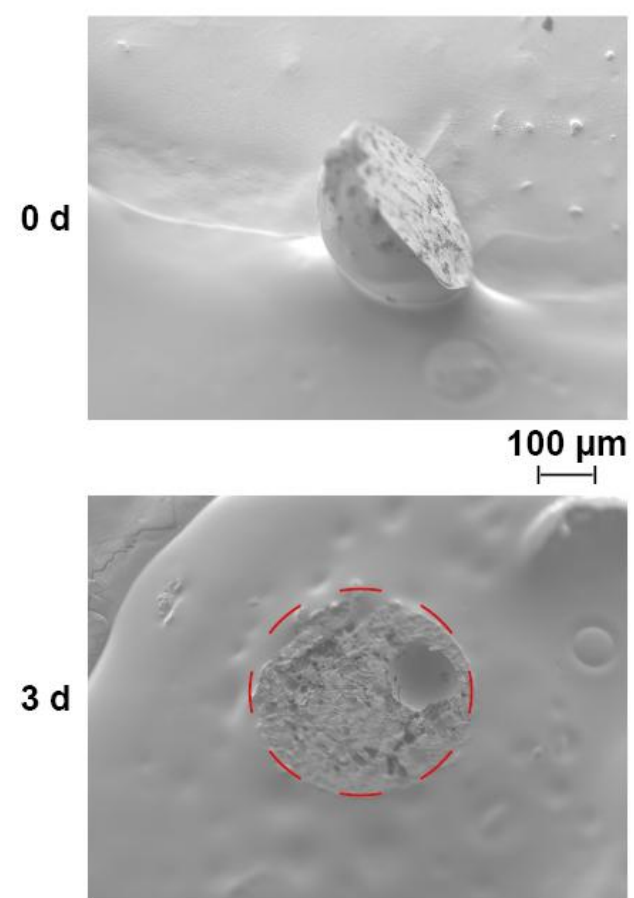

$100 \mu \mathrm{m}$

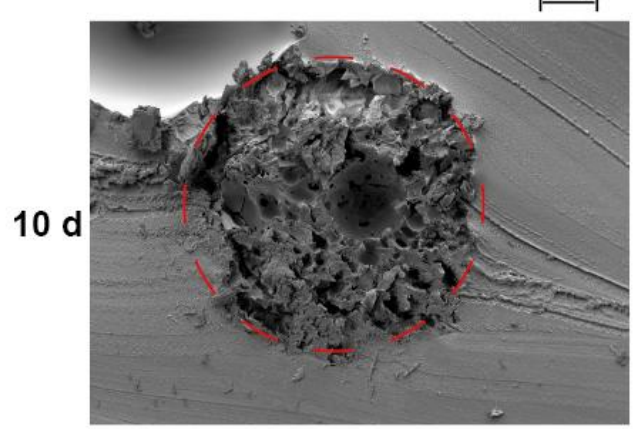

$100 \mu \mathrm{m}$

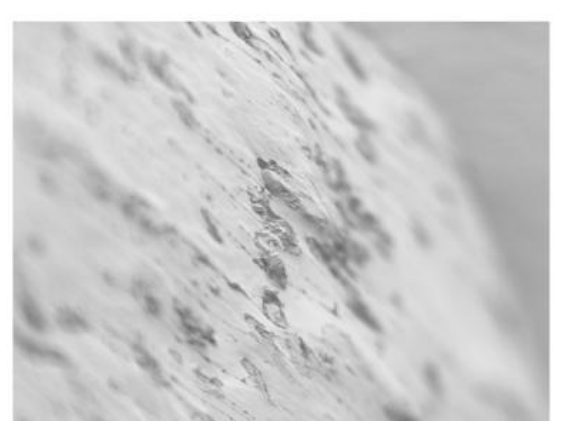

$10 \mu \mathrm{m}$

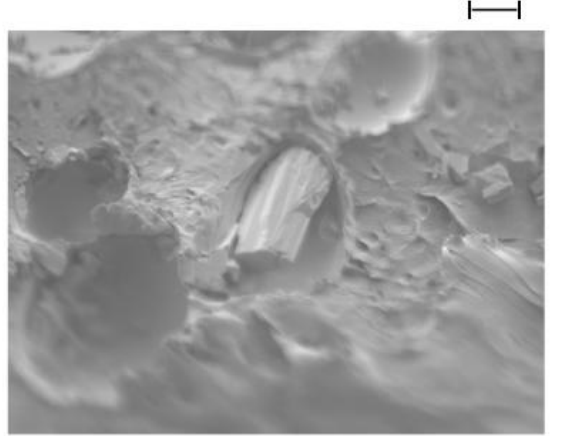

$10 \mu \mathrm{m}$

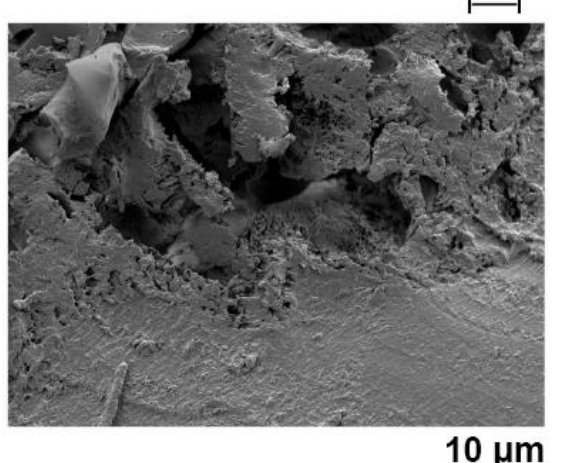

$10 \mu \mathrm{m}$

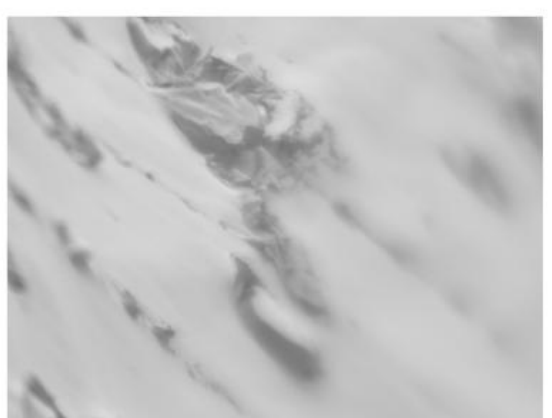

$1 \mu \mathrm{m}$

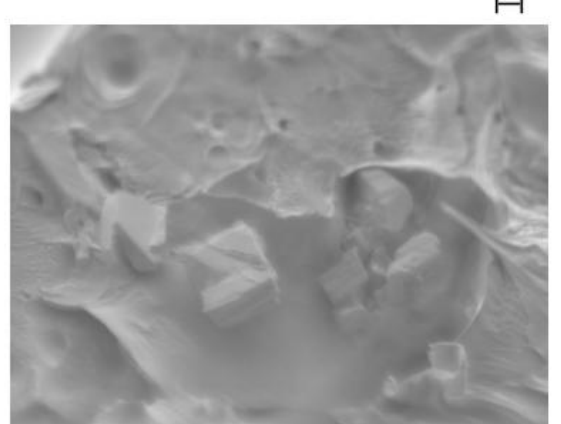

$1 \mu \mathrm{m}$

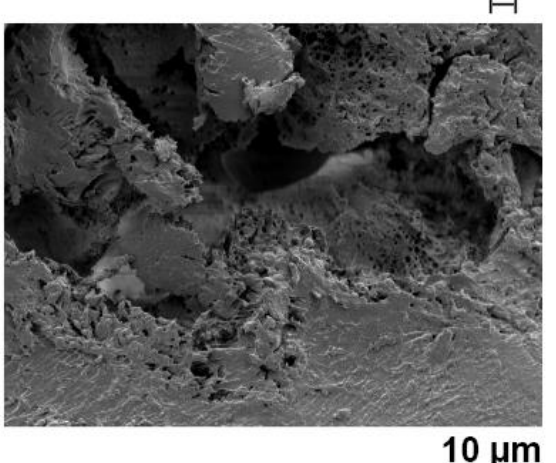

$10 \mu \mathrm{m}$
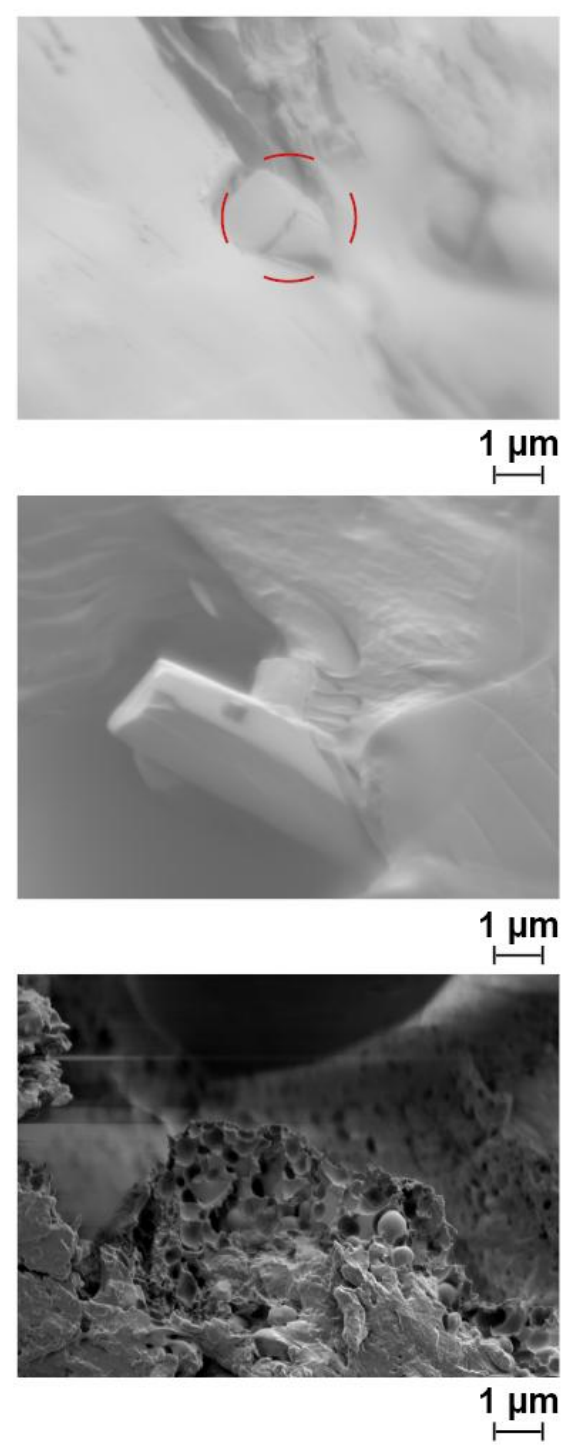

Figure 4 


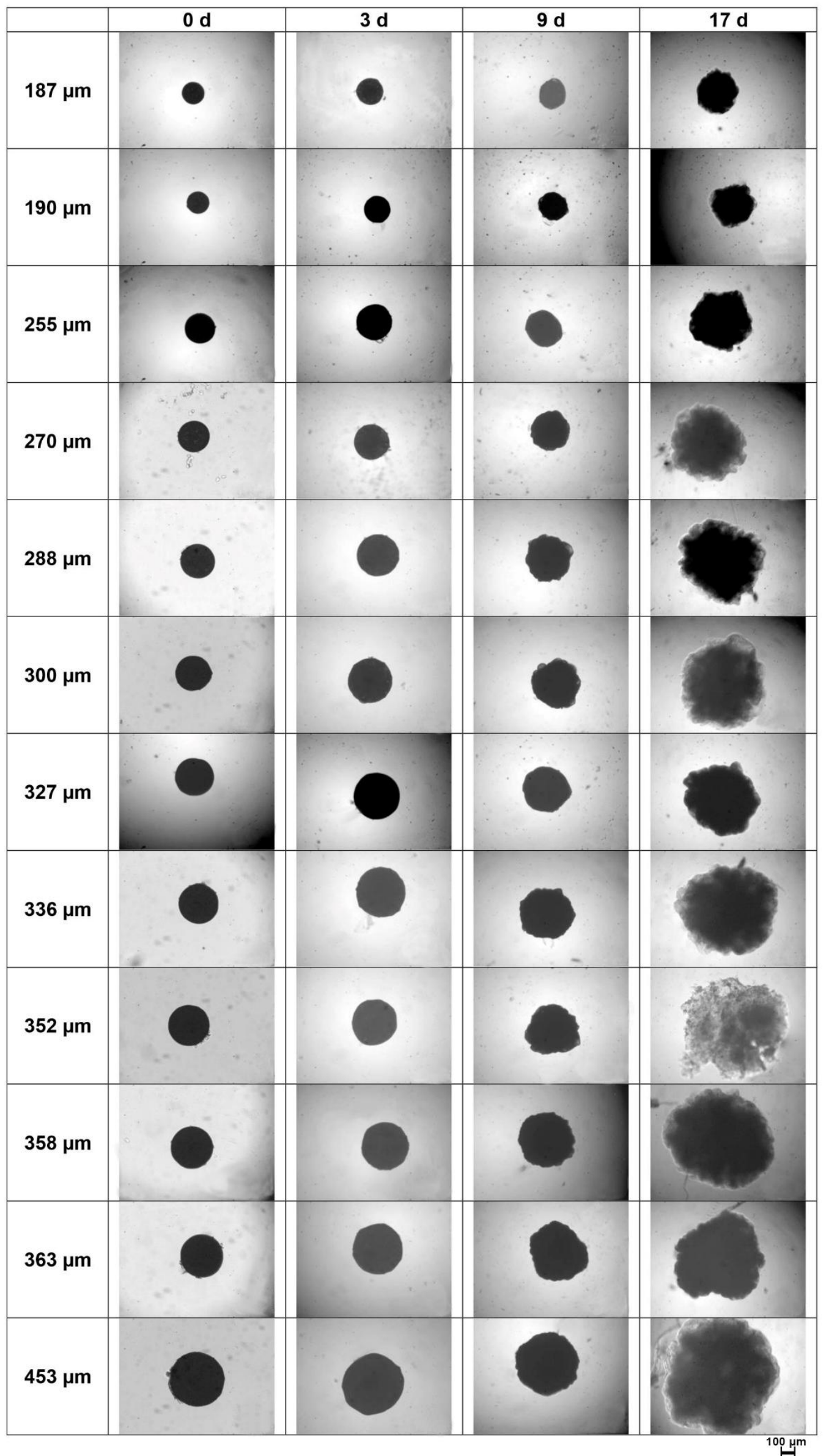

Figure 5 


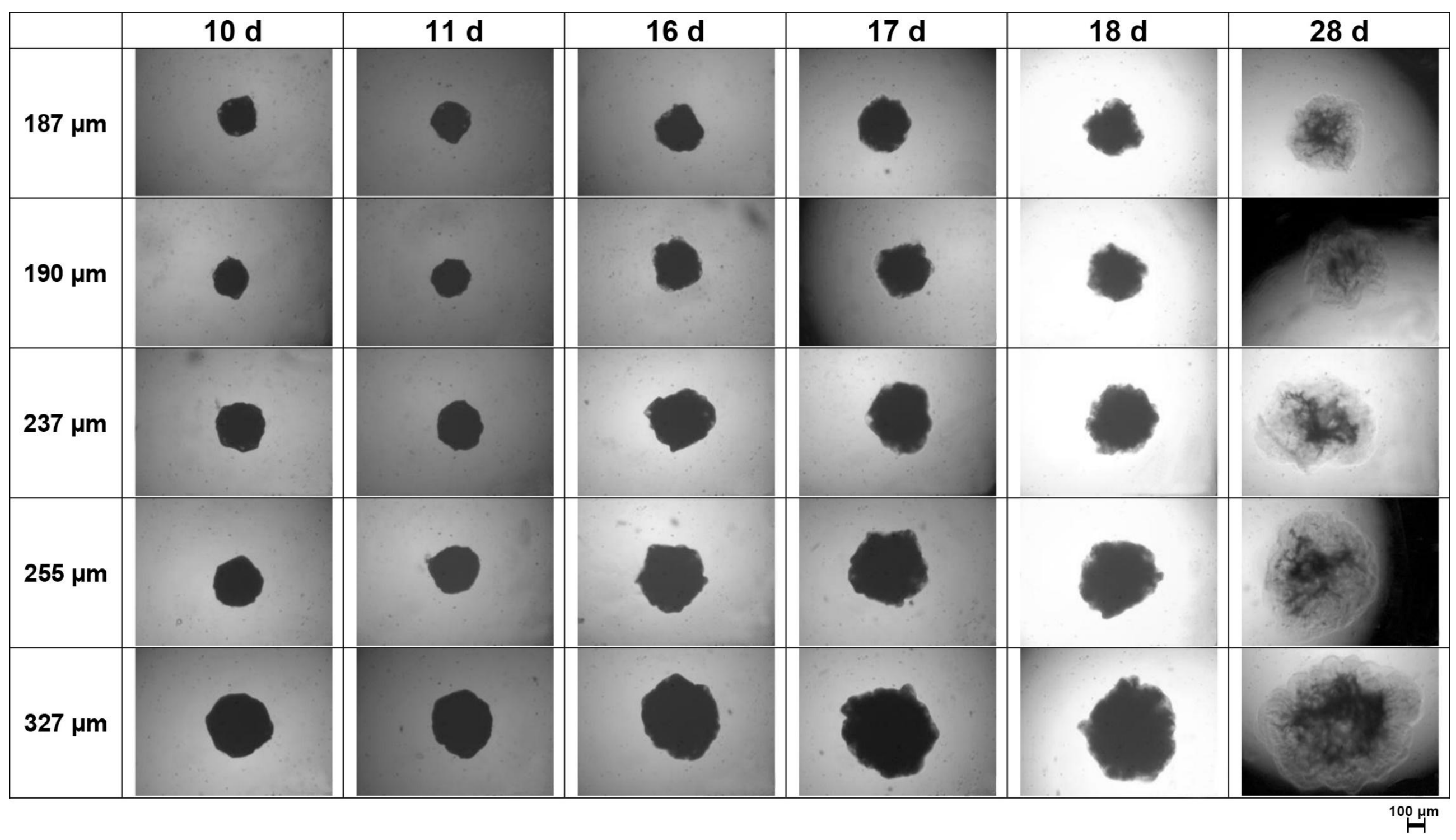

Figure 6 

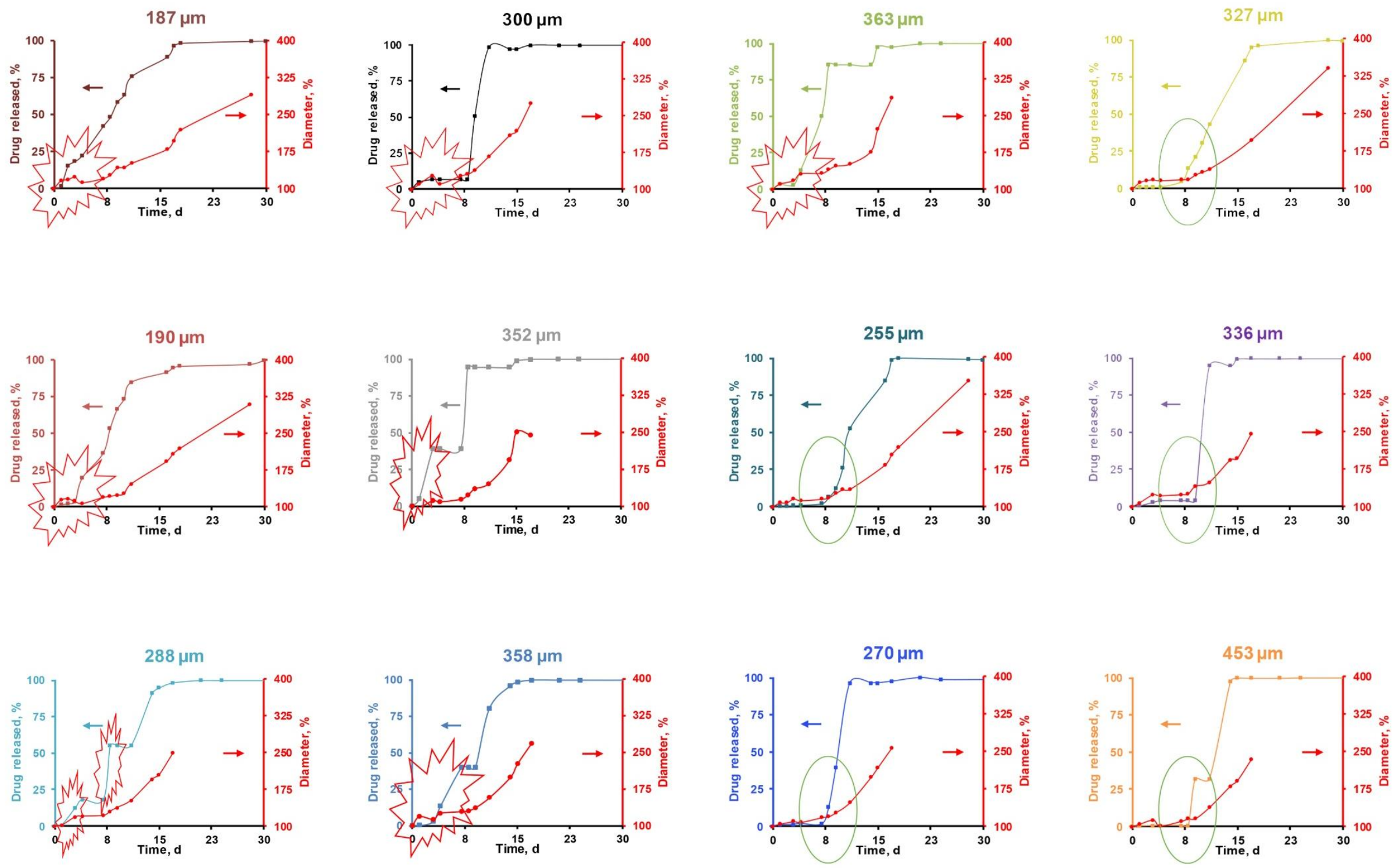

Figure 7 

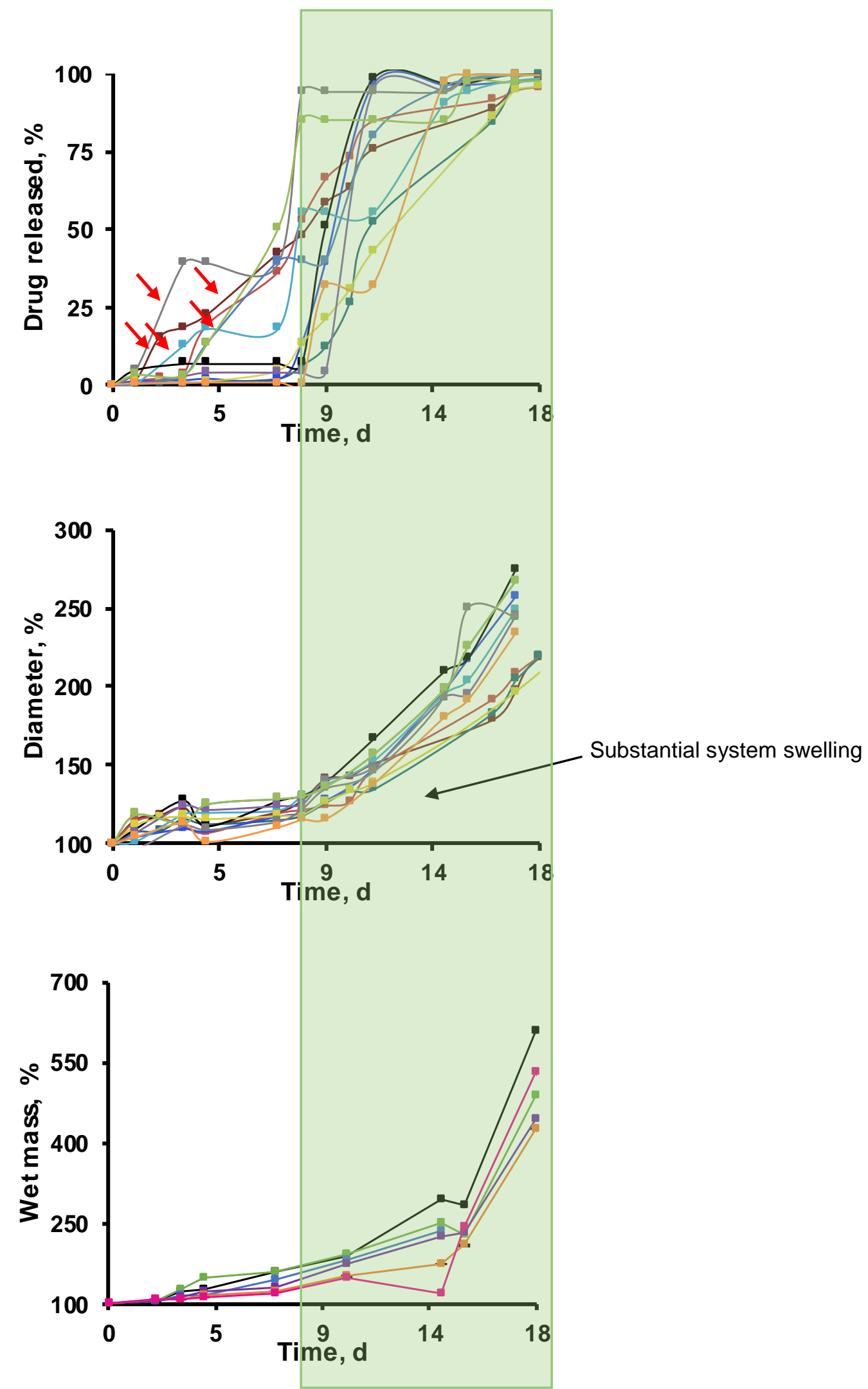

Figure 8 
$1^{\text {st }}$ Release phase ("burst release")
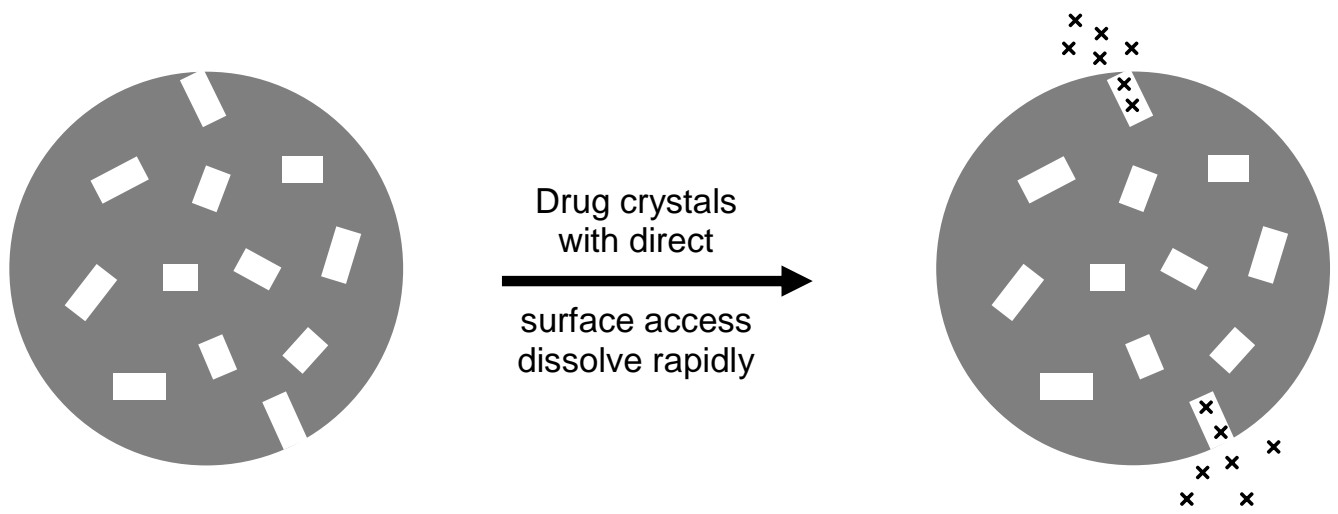

$2^{\text {nd }}$ Release phase ( constant release rate)

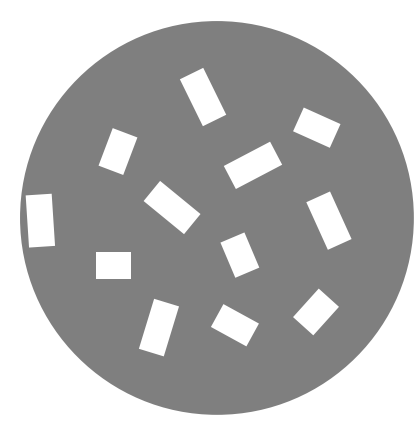

Drug crystals in swollen regions

dissolve, followed by rapid diffusion

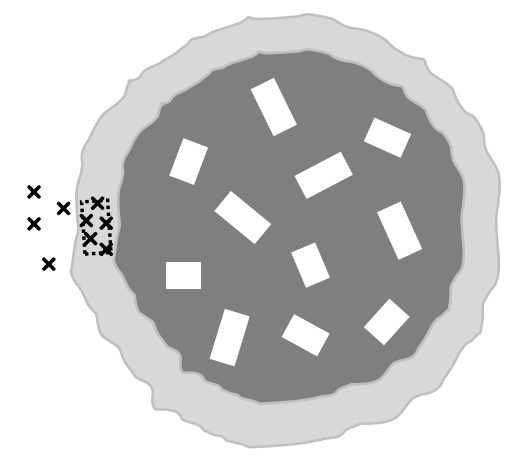

$3^{\text {rd }}$ Release phase (again rapid, leading to complete release exhaust)
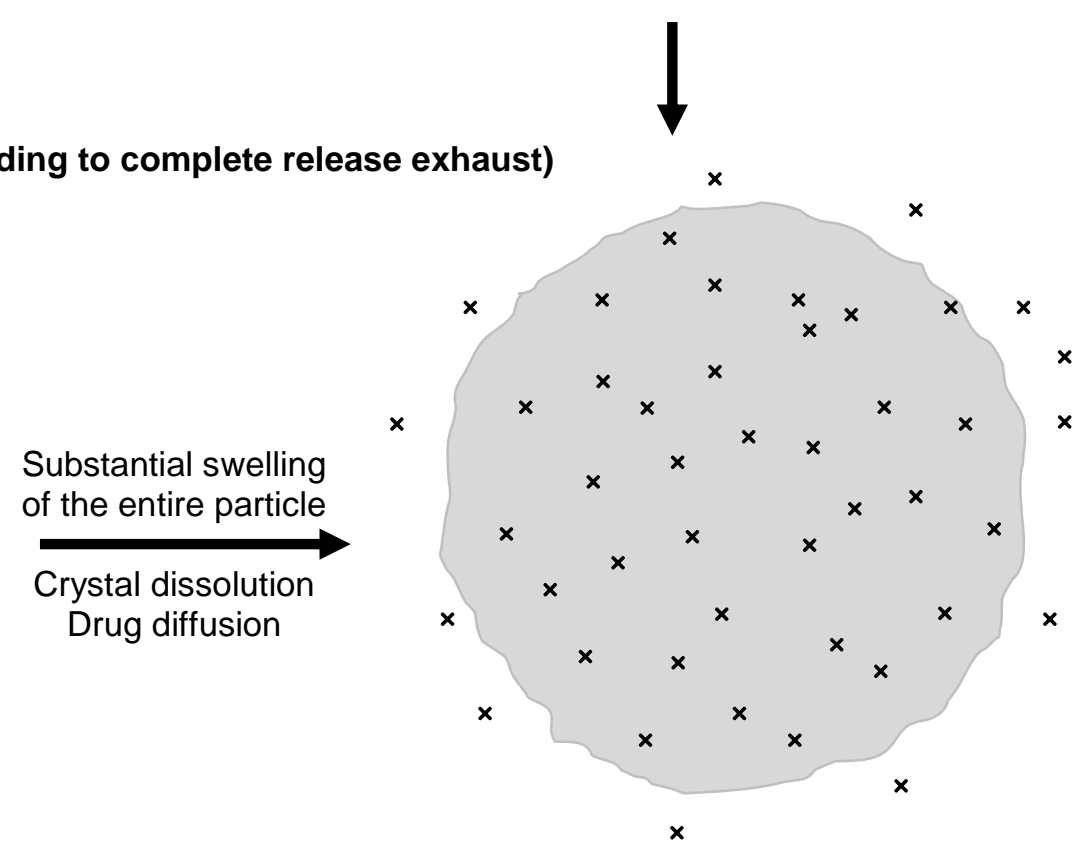

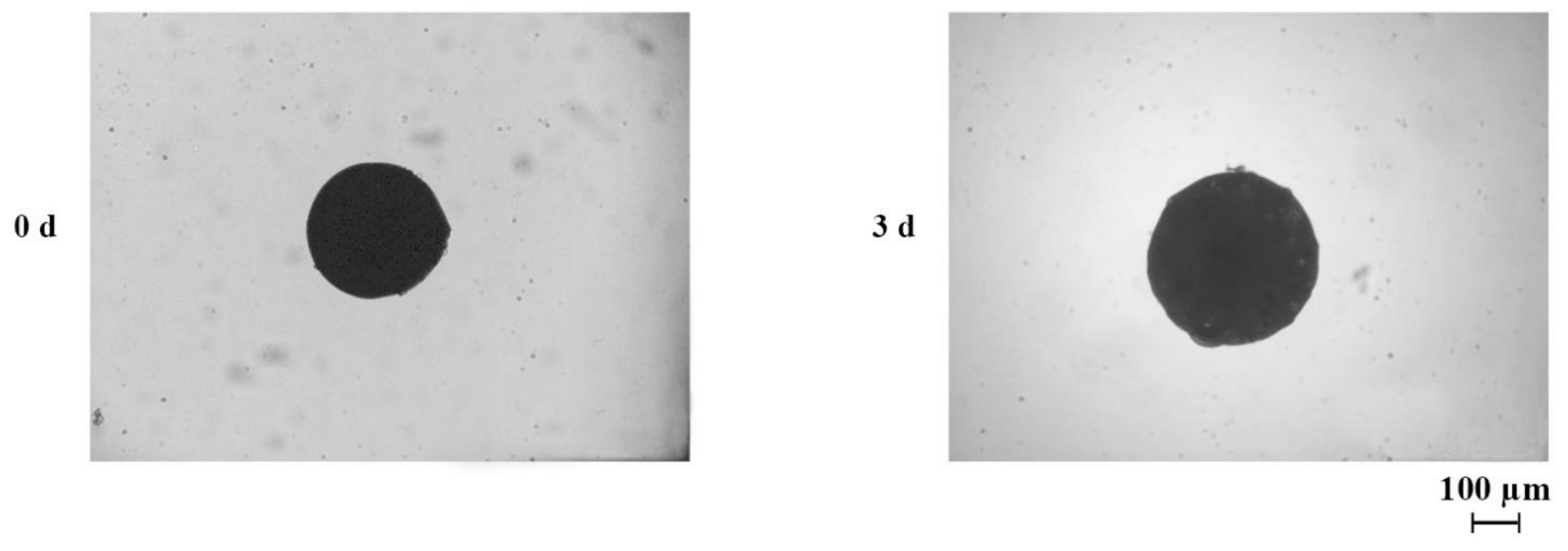

Figure 10 
a)
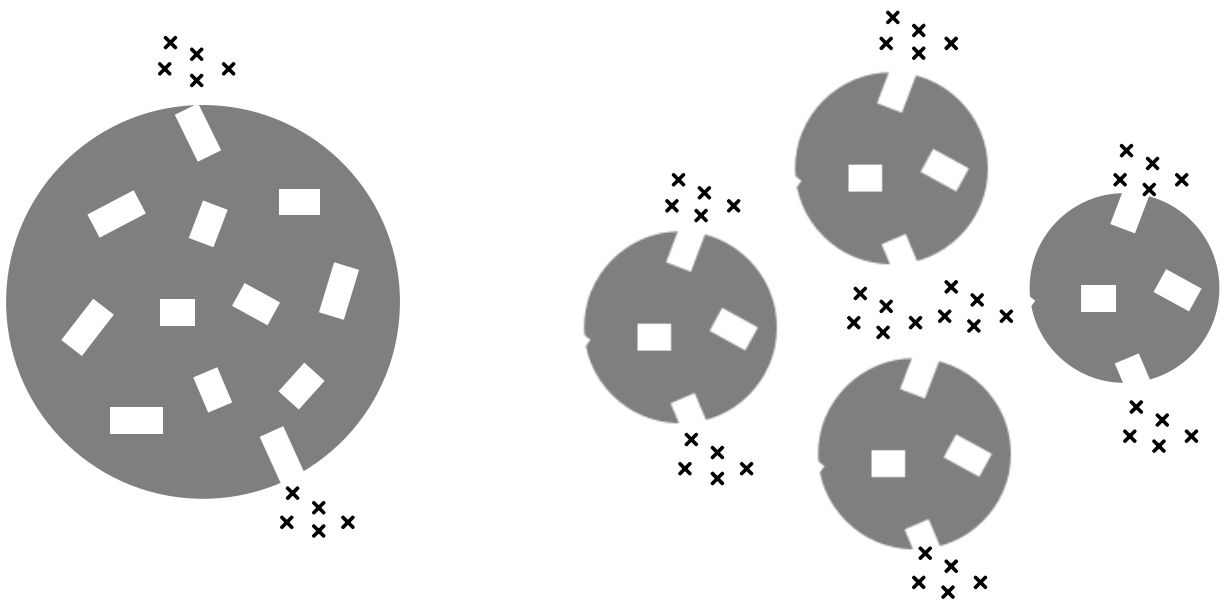

b)
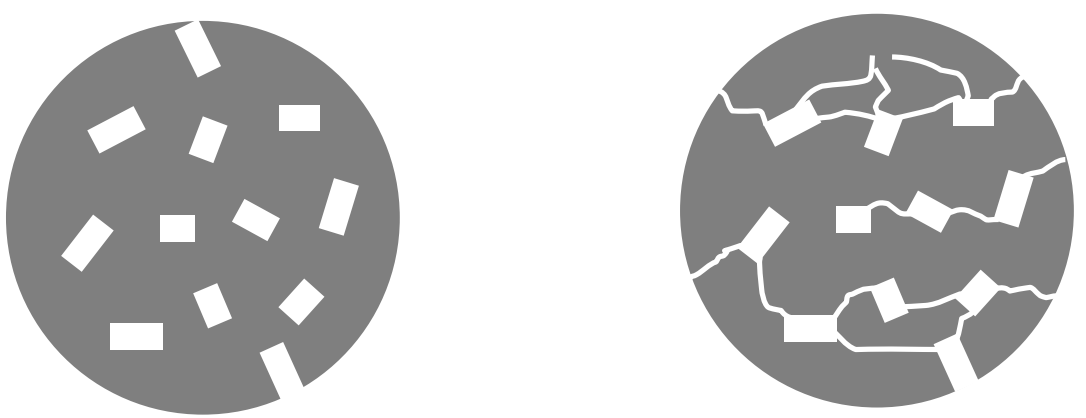

Figure 11 

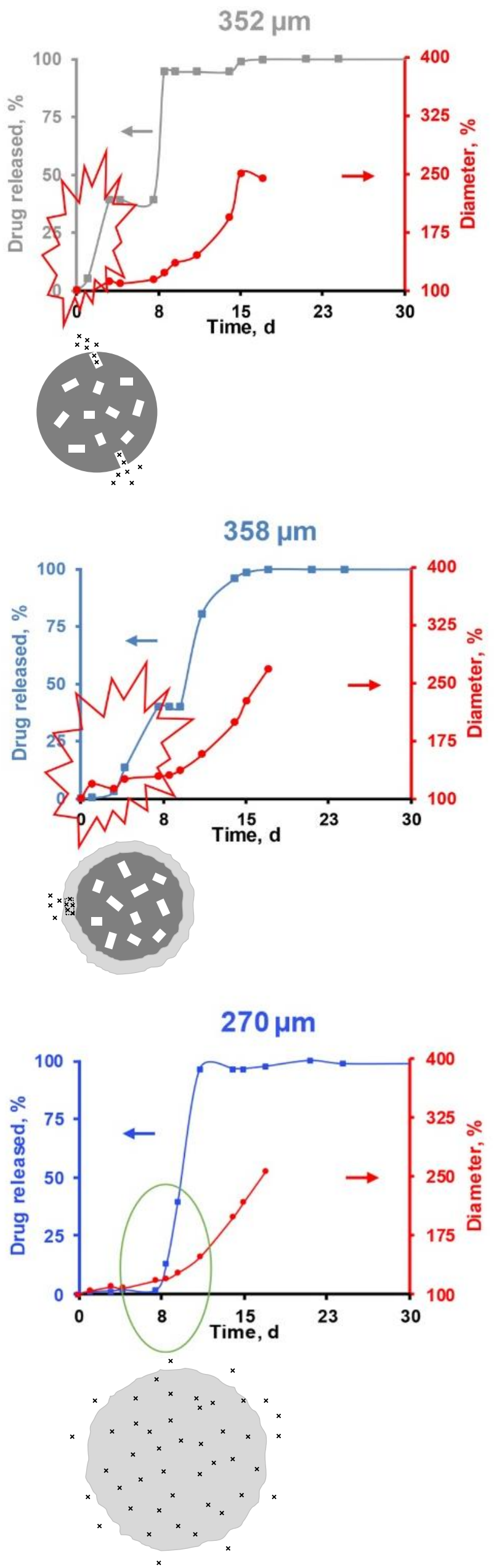

Figure 12 